\title{
Cerebrovascular dysfunction and microcirculation rarefaction precede white matter lesions in a mouse genetic model of cerebral ischemic small vessel disease
}

\author{
Anne Joutel,1,2,3 Marie Monet-Leprêtre, 1,2 Claudia Gosele,, ${ }^{4}$ Céline Baron-Menguy, 1,2 \\ Annette Hammes, ${ }^{4}$ Sabine Schmidt, ${ }^{4}$ Barbara Lemaire-Carrette,,${ }^{1,2}$ \\ Valérie Domenga, ${ }^{1,2}$ Andreas Schedl,5,6 Pierre Lacombe,1,2 and Norbert Hubner ${ }^{4}$ \\ 1INSERM U740, Paris, France. 2Université Paris 7-Denis Diderot, Faculté de Médecine, Site Villemin, Paris, France. \\ ${ }^{3}$ AP-HP, Groupe hospitalier LARIBOISIERE-FERNAND-WIDAL, Groupement hospitalier-universitaire Nord, Laboratoire de Génétique, Paris, France. \\ ${ }^{4}$ Max-Delbruck-Center for Molecular Medicine (MDC), Berlin, Germany. ${ }^{5 I N S E R M ~ U 636, ~ N i c e, ~ F r a n c e . ~}{ }^{6}$ Université de Nice/Sophia Antipolis, Nice, France.
}

\begin{abstract}
Cerebral ischemic small vessel disease (SVD) is the leading cause of vascular dementia and a major contributor to stroke in humans. Dominant mutations in NOTCH3 cause cerebral autosomal dominant arteriopathy with subcortical infarcts and leukoencephalopathy (CADASIL), a genetic archetype of cerebral ischemic SVD. Progress toward understanding the pathogenesis of this disease and developing effective therapies has been hampered by the lack of a good animal model. Here, we report the development of a mouse model for CADASIL via the introduction of a CADASIL-causing Notch3 point mutation into a large P1-derived artificial chromosome (PAC). In vivo expression of the mutated PAC transgene in the mouse reproduced the endogenous Notch3 expression pattern and main pathological features of CADASIL, including Notch3 extracellular domain aggregates and granular osmiophilic material (GOM) deposits in brain vessels, progressive white matter damage, and reduced cerebral blood flow. Mutant mice displayed attenuated myogenic responses and reduced caliber of brain arteries as well as impaired cerebrovascular autoregulation and functional hyperemia. Further, we identified a substantial reduction of white matter capillary density. These neuropathological changes occurred in the absence of either histologically detectable alterations in cerebral artery structure or blood-brain barrier breakdown. These studies provide in vivo evidence for cerebrovascular dysfunction and microcirculatory failure as key contributors to hypoperfusion and white matter damage in this genetic model of ischemic SVD.
\end{abstract}

\section{Introduction}

Ischemic cerebral small vessel disease (SVD) is the leading cause of vascular dementia and a major contributor to stroke in humans. Most ischemic cerebral SVDs involve a diffuse arteriopathy of the smaller penetrating arteries, resulting in multiple subcortical lacunar infarctions and more diffuse areas of white matter lesions radiologically referred to as leukoaraiosis (1). However, despite the importance of SVD, there are no specific treatments. This is mainly due to a poor understanding of the disease pathogenesis, although hypertension has been identified as a major risk factor, and the lack of appropriate animal models (2).

Dominant mutations in the NOTCH3 gene cause cerebral autosomal dominant arteriopathy with subcortical infarcts and leukoencephalopathy (CADASIL), a genetic archetype of nonhypertensive ischemic cerebral SVD and the most common heritable cause of stroke and vascular dementia in adults (3-5). Clinical features resemble those of nonhereditary SVD except for an earlier age of onset and an increased frequency of migraine with aura. Overall, ischemic events are present in $60 \%-85 \%$ of patients and occur between 40 and 60 years of age, cognitive impairment results in subcortical dementia between 50 and 60 years of age, and patients

Authorship note: Marie Monet-Leprêtre and Claudia Gosele contributed equally to this work.

Conflict of interest: The authors have declared that no conflict of interest exists. Citation for this article: J Clin Invest. 2010;120(2):433-445. doi:10.1172/JCI39733. become bedridden and die around 65-70 years of age $(6,7)$. Leukoaraiosis is the earliest, consistent MRI change preceding the onset of ischemic and cognitive symptoms by $10-15$ years (8). Autopsy studies in patients with CADASIL demonstrated an arteriopathy affecting primarily the cerebral small penetrating and pial arteries, that is characterized by a thickening and fibrosis of the arterial wall and prominent alterations of smooth muscle cells which eventually disappear. Distinct from other causes of SVD, vessels exhibit pathognomonic deposits of granular osmiophilic material (GOM) of unknown composition (9-11).

Notch3 encodes a transmembrane receptor, the postnatal expression of which is predominantly restricted to vascular smooth muscle cells and pericytes (12). Notch3 is initially synthesized as an approximately $280-\mathrm{kDa}$ precursor, which then undergoes proteolytic processing similar to other Notch receptors. This results in the formation of a mature heterodimeric receptor consisting of a 210-kDa extracellular domain (Notch $3^{\mathrm{ECD}}$ ) noncovalently attached to a $97-\mathrm{kDa}$ transmembrane/intracellular fragment (Notch3 ${ }^{\mathrm{TMIC}}$ ). CADASIL patients carry highly stereotyped mutations, which alter the number of cysteine residues in the extracellular domain of NOTCH3 (13-15). These mutations are associated with vascular accumulation of NOTCH $3^{\mathrm{ECD}}$ without associated NOTCH3 $3^{\mathrm{TMIC}}$ accumulation, at the plasma membrane of smooth muscle cells and pericytes in close vicinity to or within the GOM deposits (12, $16,17)$. The molecular pathways linking NOTCH3 mutations to degeneration of vascular cells are as yet incompletely understood. 
A

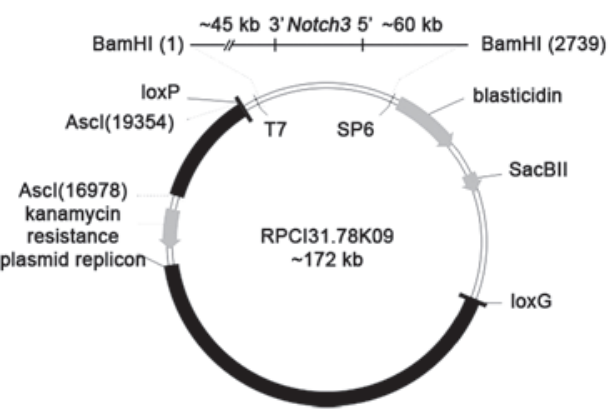

C

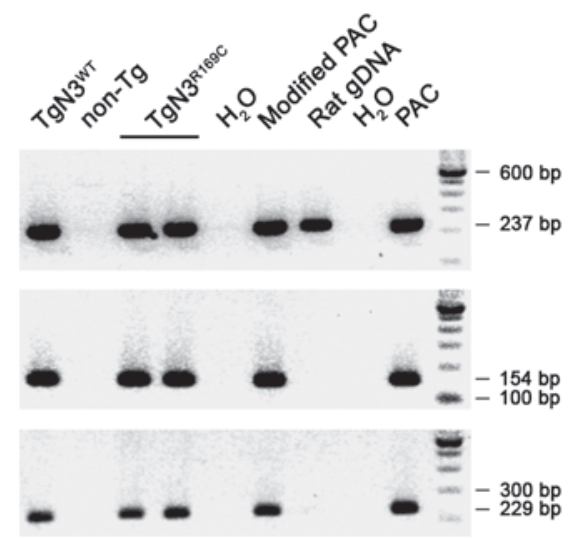

E

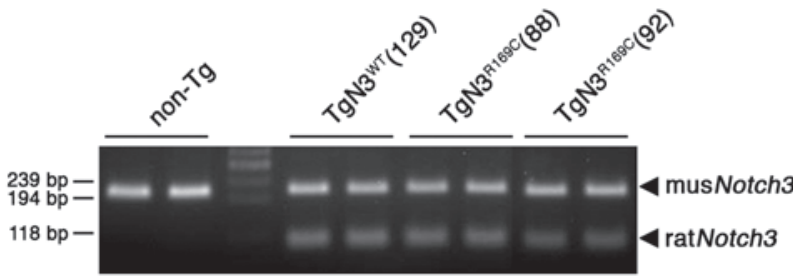

G

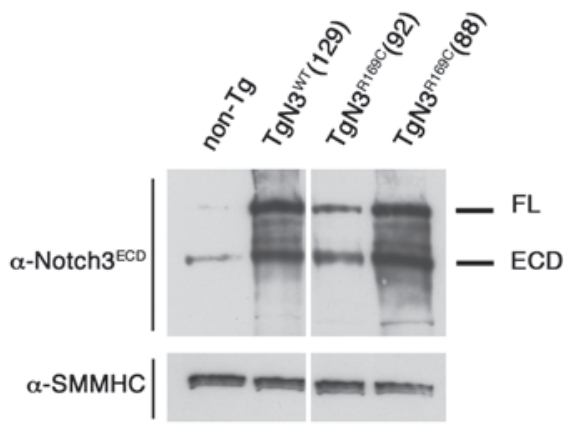

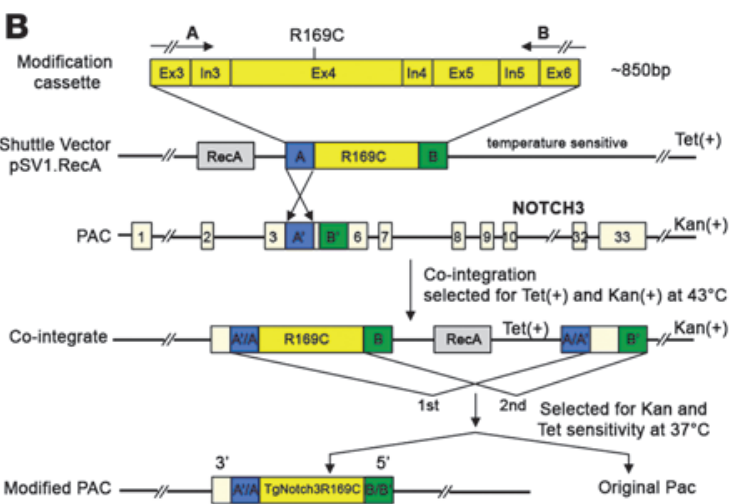

D

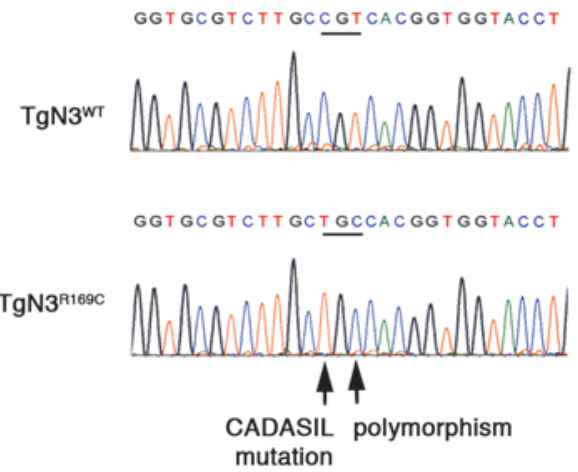

$\mathbf{F}$
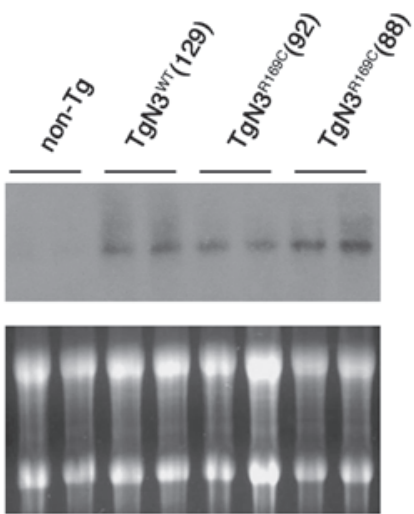


\section{Figure 1}

Generation and characterization of PAC transgenic mice expressing wild-type or CADASIL-linked R169C rat Notch3. (A) Rat PAC clone and vector map $(27,52)$. (B) Strategy for targeted PAC modification according to ref. 28. This panel was adapted from ref. 28 with permission of Nature biotechnology. The modification cassette, inserted in the shuttle vector, contains flanking rat Notch 3 genomic sequence of exons 3 and 6 on both sides of the engineered CADASIL mutation in exon 4 . Two PAC modification steps are illustrated: co-integration of the shuttle vector into the PAC (1st and 2nd) and the resolution of the co-integrant by a second homologous recombination event to eliminate the shuttle vector and other exogenous sequences leaving the modified PAC carrying a point mutation. (C) Full-length integration of wild-type and modified PACs in transgenic mice. Shown are PCR products corresponding to rat Notch3 exon 1 (top), SP6 site (middle), and T7 site (bottom) of the PAC clone. (D) Sequence analysis of the PCR product from the rat Notch3 transgene in TgNotch3WT (TgN3WT) and TgNotch33169C (TgN3R169C) mice. (E) RT-PCR assay of brain from nontransgenic, TgNotch3 ${ }^{\mathrm{WT}}$, and TgNotch ${ }^{R}{ }^{169 C}$ (lines 88 and 92) mice. PCR products were cleaved with Rsal and fractionated on agarose gel, yielding an uncleaved 206-bp fragment from endogenous mouse Notch3 mRNA and 108- and 98-bp cleaved fragments from rat Notch3 mRNA. (F) Northern blot analysis of brain from nontransgenic, TgNotch ${ }^{\mathrm{WT}}$, and TgNotch3 ${ }^{\mathrm{R} 169 \mathrm{C}}$ mice. (G) Representative immunoblots of brain lysates prepared from 1-monthold TgNotch3 ${ }^{\mathrm{R} 169 \mathrm{C}}$ (lines 88 and 92), TgNotch3 ${ }^{\mathrm{WT}}$, and nontransgenic mice probed with the $5 \mathrm{E} 1$ anti-Notch $3 \mathrm{ECD}$ antibody, which recognizes endogenous mouse and exogenous rat Notch3 proteins, and the anti$\mathrm{SMMHC}$ antibody. White lines indicate that the lanes were run on the same gel but were noncontiguous.

However, it is noteworthy that total loss of Notch3 in the mouse, although leading to structural and functional alterations of small arteries, does not cause CADASIL pathology $(18,19)$. Yet, in vitro assays as well as genetic studies in humans and mice support a model that evokes novel pathogenic roles for the mutant NOTCH3 protein, rather than compromised NOTCH3 function as the primary determinant of the CADASIL disease (20-23).

We and others have attempted to model CADASIL in the mouse, with limited success. Lundkvist and colleagues generated a mouse knockin model for the p.Arg141>Cys mutation, but these mice developed neither the vascular alterations nor the brain lesions characteristic of CADASIL (24). We produced transgenic mice that expressed human NOTCH3 cDNA with the p.Arg90>Cys or p.Cys428>Ser mutation in smooth muscle cells at levels approximately 1- to 2-fold of those of normal Notch3 levels in these cells, using the smooth muscle cell-specific SM22 $\alpha$ promoter element. Upon aging, these mice developed microscopic aggregates of NOTCH $3^{\mathrm{ECD}}$ and GOM deposits in the arteries, but they still did not develop brain lesions $(23,25)$.

The usual challenge with late-onset neurodegenerative diseases is that the short life span of the mouse is often limiting, as prolonged exposure to the mutant protein is assumed to be necessary to trigger cell dysfunction or degeneration. Conventional transgenesis is frequently hampered by low-level transgene expression and extensive position effects, since elements that are important for high-level, tissue-specific, and integration site-independent expression of a transgene, such as enhancers, locus control regions, and insulators, may reside at large distances from the gene itself (26). Here we used a P1-derived artificial chromosome-based (PACbased) transgenesis approach to overexpress Notch3 in an endogenous-like expression pattern. We report that mice transgenic for a large genomic segment harboring a CADASIL-causing Notch3 mutation develop most of the cardinal pathological features of the disease, including vascular and brain parenchyma lesions. Analysis of these mice allowed us to probe important aspects of CADASIL pathogenesis, such as the structural and functional consequences of the mutant protein on the brain vasculature. Our findings provide evidence for cerebrovascular dysfunction and microcirculatory failure as key contributors to disease initiation in this novel genetic model of ischemic cerebral SVD.

\section{Results}

Generation of wild-type and CADASIL-linked Notch3 PAC transgenic mice. For the generation of PAC transgenic mice, the rat PAC library (27) was chosen due to the great identity between the mouse and rat Notch3 gene (93\%), compared with an identity of mouse versus human Notch 3 of $84 \%$ and the good differentiation between exogenous Notch3 (rat) and endogenous Notch3 (mouse). We used the 172-kb PAC clone RPCI31.78K09, which contains the rat genomic Notch 3 locus plus about $60-\mathrm{kb}$ of $5^{\prime}$ flanking and $45-\mathrm{kb}$ of 3 ' flanking sequence (Figure 1A). We adopted a previously described strategy (28) using homologous recombination in E. coli to introduce the CADASIL amino acid substitution p.Arg169>Cys into this PAC (Figure 1B). We subsequently introduced the wild-type and modified intact large genomic DNA fragments into the germline of the mouse and established 3 heterozygous transgenic mouse lines expressing wild-type Notch3 (TgNotch $3^{\mathrm{WT}}$, line 129) or the CADASIL-causing Notch3 mutant (TgNotch3 ${ }^{\mathrm{R} 169 \mathrm{C}}$, lines 88 and 92). Results from PCR and Southern blotting supported integration of the intact full-length mutant and wild-type Notch3 PACs at a single site of insertion in all 3 established lines (lines 88, 92, and 129) (Figure 1, C and D, and data not shown).

RT-PCR amplification of a segment from Notch 3 mRNA followed by cleavage with RsaI, which permits discrimination between mouse and rat Notch3, demonstrated that Notch 3 mRNAs were transcribed from both the endogenous and exogenous Notch3 loci in all 3 transgenic lines (Figure 1E). Northern blot and Western blot analyses of brain derived from 1-month-old mice showed that total Notch3 transcript and protein, reflecting endogenous and transgene expression, were increased around 4-fold greater in lines TgNotch $3{ }^{\text {WT }}$ (line 129) and TgNotch $3^{\text {R169C }}$ (line 88) and about 2-fold greater in the line TgNotch3 ${ }^{\mathrm{R} 169 \mathrm{C}}$ (line 92) (Figure 1, F and G).

Importantly, in situ hybridization revealed an indistinguishable cellular distribution of Notch3 mRNA between Notch3 transgenic and nontransgenic brains. Specifically, predominant expression was detected in the vasculature with robust signal in arteries and capillaries. Lower expression was also observed in the cerebellum in a pattern consistent with the Bergmann astrocytes, while expression was negligible in all other cell types (Figure 2), as previously reported $(12,29)$. Consistent with the northern blot data, Notch3 expression level was stronger in transgenic compared with nontransgenic brains and was comparable between TgNotch ${ }^{\text {WT }}$ (line 129) and TgNotch $3^{\mathrm{R} 169 \mathrm{C}}$ (line 88) mice. Therefore, the higherexpression mutant line (line 88) was used for further experimentations unless otherwise specified.

GOM deposits in TgNotch3 $3^{R 169 C}$ mice. Presence of GOM deposits, the vascular signature of CADASIL, was assessed in the brain of TgNotch $3^{\mathrm{R} 169 \mathrm{C}}, \mathrm{TgNotch} 3^{\mathrm{WT}}$, and nontransgenic mice between 1 and 20 months of age by transmission electron microscopy analysis. GOM deposits were first detected in pial arteries of mutant mice at 5 months of age; they were located within the basement 

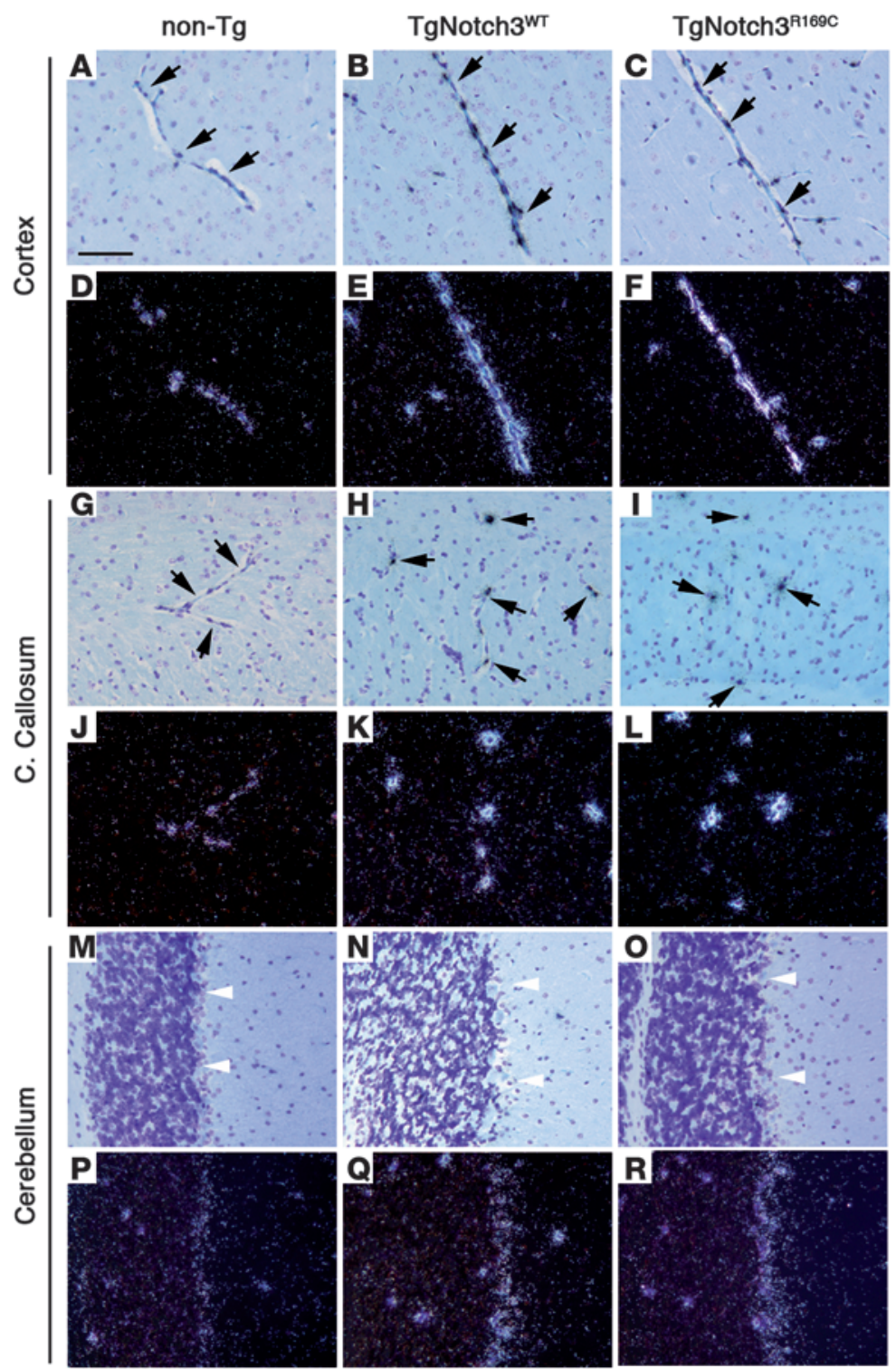

Figure 2

Transgenic mice overexpress Notch3 in an endogenous-like expression pattern. In situ hybridization with a Notch3 antisense riboprobe on sections of the cortex $(\mathbf{A}-\mathbf{F})$, corpus callosum $(\mathbf{G}-\mathbf{L})$, and cerebellum $(\mathbf{M}-\mathbf{R})$ from 1-month-old nontransgenic, TgNotch3 ${ }^{W T}$ (line 129), and TgNotch ${ }^{R 169 C}$ (line 88) mice. Bright- and dark-field images are shown on the upper and lower panel of each structure, respectively. In the cortex and corpus callosum, Notch3 expression was essentially detected in the capillaries (black arrows), with a robust signal in transgenic mice and lesser signal in non-transgenic mice. Weaker expression was also detected in the Bergmann glial cells of the cerebellum (white arrowheads) in both nontransgenic and transgenic mice, with higher expression in the latter. Note that brains were formalin fixed by immersion without prior transcardiac perfusion of the mice. Scale bar: $60 \mu \mathrm{m}$.

membrane of smooth muscle cells and were similar to those found in CADASIL patients (Figure 3A). GOM deposits further increased in number with age and became widely distributed throughout the brain arteries and capillaries by 10-12 months of age. Importantly, they were never detected in control nontransgenic and TgNotch $3^{\mathrm{WT}}$ mice up to 20 months of age (data not shown).
Microscopic aggregates of Notch $3^{E C D}$ in TgNotch $3^{R 169 C}$ mice. We compared the distribution of Notch3 polypeptides in the brains of TgNotch $3^{\mathrm{R} 169 \mathrm{C}}$ mice, TgNotch $3^{\mathrm{WT}}$ mice, and nontransgenic littermates between 1 and 20 months using antibodies specific for extracellular Notch3 (5E1) and intracellular Notch3 (Bc4). We also used antibodies to basement membrane proteins, perlecan and collagen IV, to label brain vessels and smooth muscle $\alpha$-actin antibody to distinguish arteries from capillaries. Notch3 immunostaining was restricted to the vasculature in both wild-type and mutant transgenic mice, and endogenous Notch3 in nontransgenic mice was barely detectable in this assay (Supplemental Figure 1; supplemental material available online with this article; doi:10.1172/JCI39733DS1).

We found an onset of the characteristic Notch $3^{\mathrm{ECD}}$ aggregation, without associated accumulation of Notch $3^{\text {TMIC }}$, in the brain arteries of TgNotch $3^{\text {R169C }}$ mice at 1-2 months of age. Specifically, mutant arteries showed intense granular 5E1 staining outlining smooth muscle cells, while those in TgNotch $3^{\mathrm{WT}}$ mice exhibited diffuse faint 5E1 staining. Notably, smooth muscle cells in TgNotch $3^{\mathrm{R} 169 \mathrm{C}}$ and TgNotch $3^{\mathrm{WT}}$ mice showed similar diffuse labeling with Bc4 (Figure 3, B-G). In contrast, at 1-2 months of age, brain capillaries exhibited distinct Notch3 labeling, although comparable between TgNotch $3^{\mathrm{R} 169 \mathrm{C}}$ and TgNotch $3^{\mathrm{WT}}$ mice (Figure $3, \mathrm{H}$ and $\mathrm{K}$ ). Particularly, in both TgNotch $3^{\mathrm{R} 169 \mathrm{C}}$ and TgNotch $3^{\mathrm{WT}}$ mice, pericytes exhibited large perinuclear inclusions strongly labeled by 5E1 and Bc4 (Figure 3, I and L) that closely resembled those seen in cultured cells overloaded with Notch3 proteins (ref. 30 and our unpublished observations). Remarkably, while these cytoplasmic inclusions gradually decreased in number with aging in TgNotch $3^{\mathrm{R} 169 \mathrm{C}}$ mice and to a lesser extent in TgNotch ${ }^{\mathrm{WT}}$ mice, intense dot-like 5E1 staining outlining capillaries, associated with a faint $\mathrm{Bc} 4$ staining, appeared in mutant but not wild-type capillaries and further increased in number (Figure 3 , J and $\mathrm{M}$ ). At 10-12 months of age, cytoplasmic inclusions had almost disappeared in mutant brain vessels and nearly all capillaries exhibited microscopic Notch $3^{\mathrm{ECD}}$ aggregates. We always noticed that Notch $3^{\mathrm{ECD}}$ aggregates were strictly restricted to the vasculature. Moreover, Notch $3^{\mathrm{ECD}}$ aggregates were never detected in TgNotch $3^{\mathrm{WT}}$ mice up to 20 months of age (Supplemental Figure 2).

Progressive white matter lesions in TgNotch $3^{R 169 C}$ mice. TgNotch $3^{\mathrm{R} 169 \mathrm{C}}$ mice had life spans comparable to control mice up to 24 months of age and developed neither acute nor chronic motor deficits. Nevertheless, mutant mice exhibited, at approximately 18-20 months, extensive cerebral white matter damage. Hematoxylin and eosin staining of brain sections revealed numerous vacuoles in the white matter of TgNotch3 ${ }^{\mathrm{R} 169 \mathrm{C}}$ mice, particularly in the corpus callosum, anterior commissura, fimbria of hippocampus, internal capsula, and white matter bundles of the striatum, whereas the neocortex appeared histologically intact (Figure 4G and Supplemental Figure 3). Moreover, Klüver-Barrera Luxol fast blue staining showed reduced luxol staining of white matter bundles and loss 

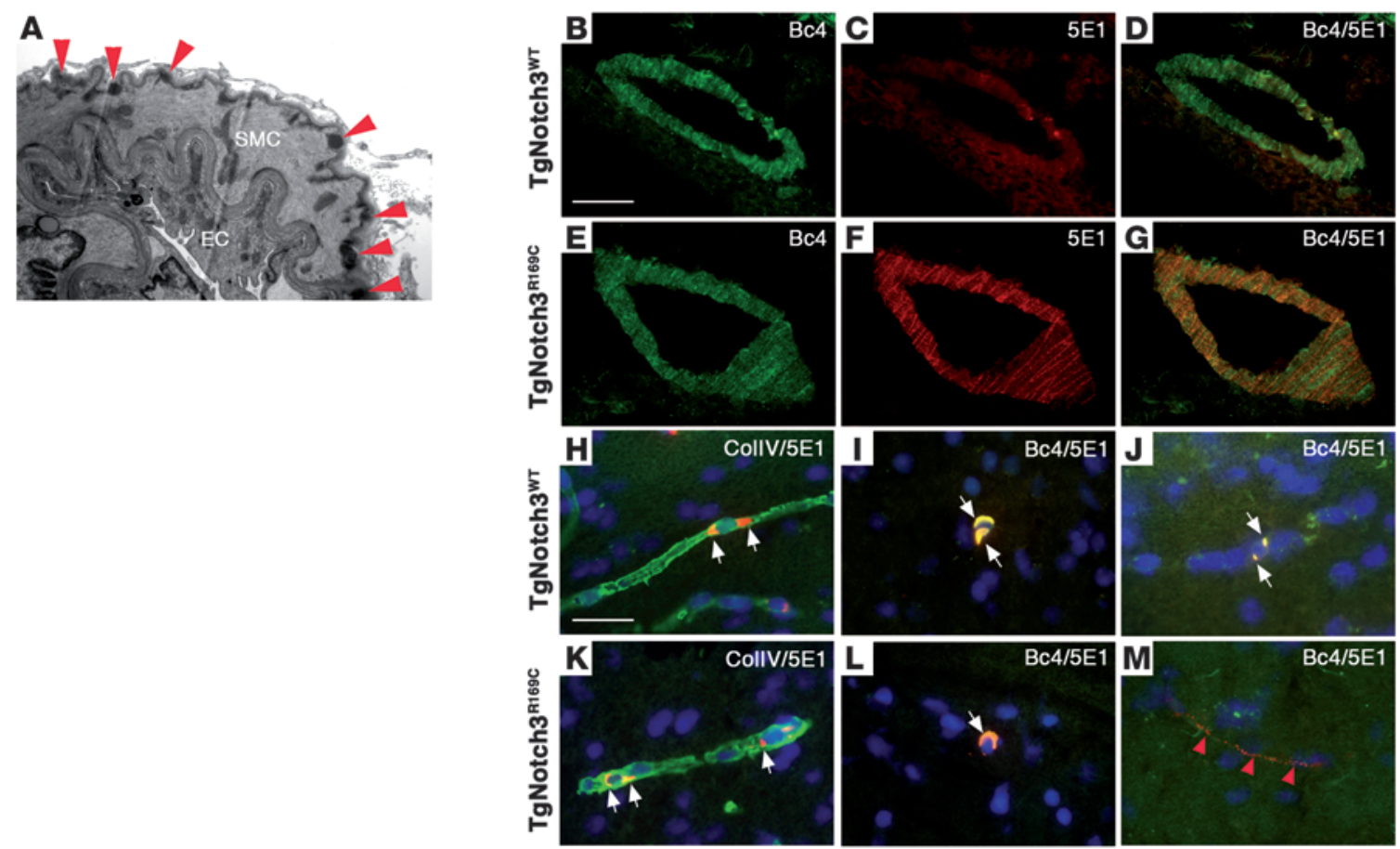

\section{Figure 3}

Notch3 ${ }^{E C D}$ aggregates and GOM deposits in brain arteries and capillaries of TgNotch3 ${ }^{\mathrm{R} 169 \mathrm{C}}$ mice. (A) Electron micrograph of a pial artery from a 5-month-old TgNotch ${ }^{\mathrm{R} 169 \mathrm{C}}$ mouse demonstrating abundant GOM deposits (red arrowheads) within the basement membrane of smooth muscle cells. (B-G) Brain arteries from 2-month-old TgNotch3 ${ }^{\mathrm{R} 169 \mathrm{C}}$ and TgNotch3 ${ }^{\mathrm{WT}}$ mice were stained with Notch3 antibodies specific to the intracellular (Bc4, green) or extracellular (5E1, red) domain. Microscopic aggregates of Notch3ECD are shown in mutant artery. $(\mathbf{H}, \mathbf{I}, \mathbf{K}$, and $\mathbf{L}$ ) Brain sections of 2-month-old TgNotch3 ${ }^{\mathrm{WT}}$ and TgNotch3 ${ }^{\mathrm{R} 169 \mathrm{C}}$ were double labeled with antibodies to Notch3 extracellular domain (5E1, red) and collagen IV (CollV, green) (H and K) or double labeled with Bc4 (green) and 5E1 (red) Notch3 antibodies (I and L). Nuclei were stained by DAPI (blue). Perinuclear inclusions, strongly labeled by Notch3 intracellular and extracellular antibodies, were seen in wild-type and mutant capillaries (white arrows). ( $\mathbf{J}$ and $\mathbf{M})$ Brain sections of 12-month-old TgNotch3WT $(\mathbf{J})$ and TgNotch3R169C (M) were double labeled with Bc4 (green) and 5E1 (red) Notch3 antibodies. Shown are dot-like Notch3ECD aggregates unstained by the Bc4 antibody in the mutant capillary (red arrowheads), while wildtype capillary exhibited discrete perinuclear inclusions labeled by Notch3 intracellular and extracellular antibodies (white arrows). Scale bars: $1 \mu \mathrm{m}(\mathbf{A}), 50 \mu \mathrm{m}(\mathbf{B}-\mathbf{G})$ and $30 \mu \mathrm{m}(\mathbf{H}-\mathbf{M})$.

of compact myelin with disorganized fibers. In contrast, control TgNotch $3^{\mathrm{WT}}$ and nontransgenic mice showed nearly intact brain parenchyma (Figure 4, A-G, and Supplemental Figure 4).

We probed mouse brains with antibodies to glial fibrillary acidic protein (GFAP). Markedly increased GFAP staining was observed in the cerebral white matter in 20 -month-old TgNotch $3^{\mathrm{R} 169 \mathrm{C}}$ mice, characterized by thickened and branched astroglial processes, whereas age-matched TgNotch $3{ }^{\mathrm{WT}}$ mice showed minimal staining (Figure 4, H-N, and Supplemental Figure 4). The distribution of astrogliosis corresponded with that of vacuoles and reduced luxol staining. Although brain parenchyma appeared histologically intact in younger TgNotch $3^{\mathrm{R} 169 \mathrm{C}}$ mice (Supplemental Figure 5 , A and B), these mice showed by 12 months of age significantly increased GFAP staining, particularly in the corpus callosum and internal capsula, as compared with age-matched TgNotch $3^{\mathrm{WT}}$ mice (Supplemental Figure 5, C and D).

Decreased resting cerebral blood flow in TgNotch $3^{R 169 C}$ mice. A reduction of cerebral blood flow (CBF) has been documented in the cortex and white matter in CADASIL patients using PET (31), SPECT (32), and MRI bolus tracking (33). We measured resting CBF in awake $\mathrm{TgNotch} 3^{\mathrm{R} 169 \mathrm{C}}$ mice and control TgNotch $3^{\mathrm{WT}}$ and nontransgenic littermate mice by quantitative autoradiography using a diffusible radiolabeled tracer, which provides a measure of local tissue perfusion through brain microvascularization (Supplemental Figure 6 and Supplemental Table 1). At 18-20 months of age, we found highly significant $16.0 \% \pm 1.0 \%$ reductions in blood flow throughout the cerebral white matter in TgNotch $3^{\mathrm{R} 169 \mathrm{C}}$ mice compared with age-matched TgNotch $3^{\mathrm{WT}}$ and nontransgenic mice. These reductions ranged from $10 \%$ in the internal capsula to $19 \%$ in the corpus callosum. There were also significant $12.5 \% \pm 0.4 \%$ reductions in blood flow in the normal-appearing gray matter in mutant mice, ranging from $11 \%$ in the thalamus to $15 \%$ in the cortex (Figure 5). Significant reductions (5\%-8\%) in CBF were detectable in the gray matter of TgNotch $3^{\mathrm{R} 169 \mathrm{C}}$ mice as early as $11-12$ months of age, while white matter blood flow values, at this age, did not significantly differ between TgNotch $3^{\text {R169C }}$ and TgNotch3 ${ }^{\text {WT }}$ mice (Supplemental Figure 7).

Preserved arterial structure but rarefied white matter capillaries are found in TgNotch $3^{R 169 C}$ mice. To investigate the underlying mechanisms of white matter injury and reduced CBF, we first examined whether mutant Notch3 altered the structure of cerebral blood vessels. Despite the significant burden of Notch $3^{\mathrm{ECD}}$ accumulation in the pial and smaller penetrating arteries of 20-month-old TgNotch $3^{\text {R169C }}$ mice (Supplemental Figure 2), Masson's trichrome staining and high-resolution microscopy of semi-thin sections did not reveal overt changes in the vessel wall structure (Figure 6A and 

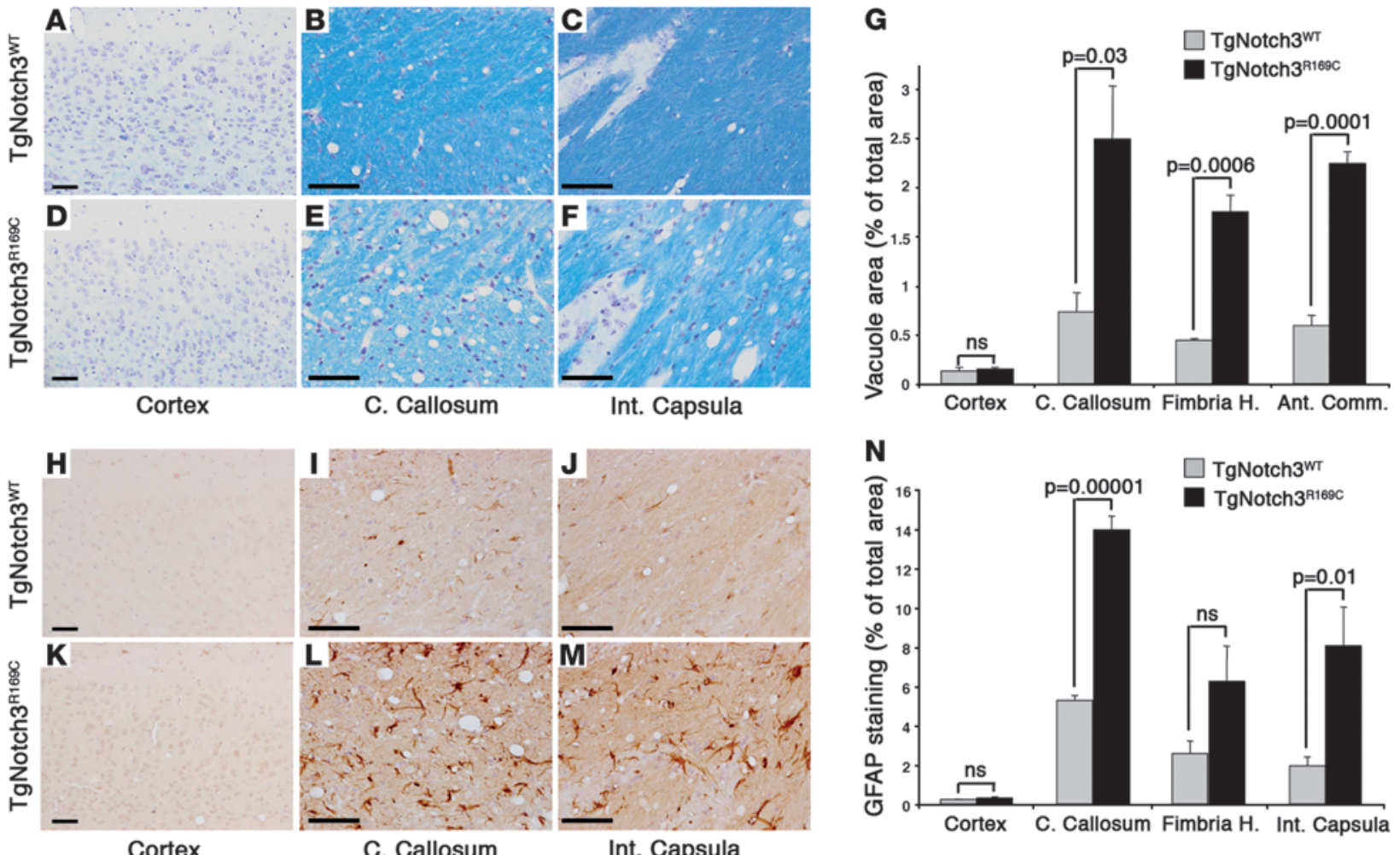

Figure 4

White matter lesions and astrogliosis in aged TgNotch3R169C mice. (A-F) Klüver-Barrera Luxol fast blue staining in the cortex, corpus callosum, and internal capsula of 20-month-old TgNotch $3^{\mathrm{WT}}$ and $\mathrm{TgNotch} 3^{\mathrm{R} 169 \mathrm{C}}$ mice revealing vacuolization in white matter bundles as well as pallor and disorganization of the myelin in mutant mice. (G) Quantification of the vacuole area in the cortex, corpus callosum, fimbria of hippocampus, and anterior commissural (Ant. Comm.) of 20-month-old TgNotch3R169C compared with TgNotch3WT mice $(n=3-4$ mice per group). (H-M) GFAP staining in the cortex, corpus callosum, and internal capsula of 20-month-old TgNotch ${ }^{W T}$ and TgNotch $3^{\text {R } 169 \mathrm{C}}$ mice showing astrogliosis in the white matter bundles of TgNotch3 ${ }^{\text {R169C }}$ mice. (N) Quantification of astrogliosis in the indicated structures of 20-month-old TgNotch3 ${ }^{\text {R169C }}$ mice compared with TgNotch3 ${ }^{\mathrm{WT}}$ mice ( $n=4$ mice per group). Scale bars: $70 \mu \mathrm{m}$.

data not shown). Additionally, smooth muscle myosin heavy chain immunolabeling showed no evidence of smooth muscle cell loss in these arteries (Figure 6B).

To assess whether mutant Notch3 impaired the brain microcirculation we quantified the mean total capillary length in TgNotch $3^{\text {R169C }}$ and TgNotch $3^{\text {WT }}$ brains. As representative brain regions without and with progressive histological alterations in TgNotch $3^{\mathrm{R} 169 \mathrm{C}}$ mice, we examined the neocortex and the corpus callosum, respectively. In mice 5 months old, we found a trend toward a decline of capillary length in the corpus callosum of TgNotch $3^{\text {R169C }}$ mice compared with age-matched TgNotch $3^{\text {WT }}$ mice. This capillary length reduction reached statistical significance by 12 months of age, contemporaneous to astrogliosis appearance but prior to white matter degeneration. Reduction was further amplified in 20-month-old mutant mice (Figure 6D). Remarkably, the mean total capillary length was comparable in the cortex of TgNotch $3^{\mathrm{R} 169 \mathrm{C}}$ and TgNotch ${ }^{\mathrm{WT}}$ mice, although the density of Notch $3^{\mathrm{ECD}}$ aggregates appeared similar between gray and white matter capillaries (Figure 6, C and D).

Preserved integrity of blood-brain barrier in TgNotch $3^{R 169 C}$ mice. We next examined the blood-brain barrier (BBB), since BBB disruption has been implicated in the pathogenesis of ischemic white matter changes $(34,35)$. We examined ultrastructural features of capillaries in the neocortex, corpus callosum, and internal capsula. Electron microsco- py analysis was performed at 12 months of age contemporaneously to astrogliosis but prior to white matter degeneration. Supplemental Figure 8A (and data not shown) shows that capillaries appeared ultrastructurally intact in TgNotch $3^{\mathrm{R} 169 \mathrm{C}}$ mice. Specifically, endothelial cells exhibited intact tight junctions and were surrounded by a thin basement membrane wrapping thin pericyte profiles and astrocytic foot processes closely apposed to the basement membrane.

We also regionally assess the BBB permeability to macromolecules. Mice were injected with a Dextran fluorescent tracer having the same size as albumin, which is widely used for investigation of BBB leakage (36). Importantly, both gray and white matter blood vessels in the TgNotch $3^{\mathrm{R} 169 \mathrm{C}}$ and TgNotch $3^{\mathrm{WT}}$ mice similarly retained the $70-\mathrm{kDa}$ fluorescent tracer within the lumen (Supplemental Figure 8, B and C, and data not shown).

Impaired cerebrovascular function in TgNotch $3^{R 169 C}$ mice. Next, we investigated whether mutant Notch 3 altered 2 major functional properties of the cerebrovasculature, i.e., CBF autoregulation and functional hyperemia (37). Mice were investigated at 5-6 months of age, when histological alterations are not yet detectable in the brain parenchyma.

Cerebrovascular autoregulation counteracts the effects of normal fluctuations in arterial blood pressure occurring during daily activities, that may lead to potentially deleterious increases or decreases in $\mathrm{CBF}$. Thus, cerebral arteries dilate when blood pressure decreases 


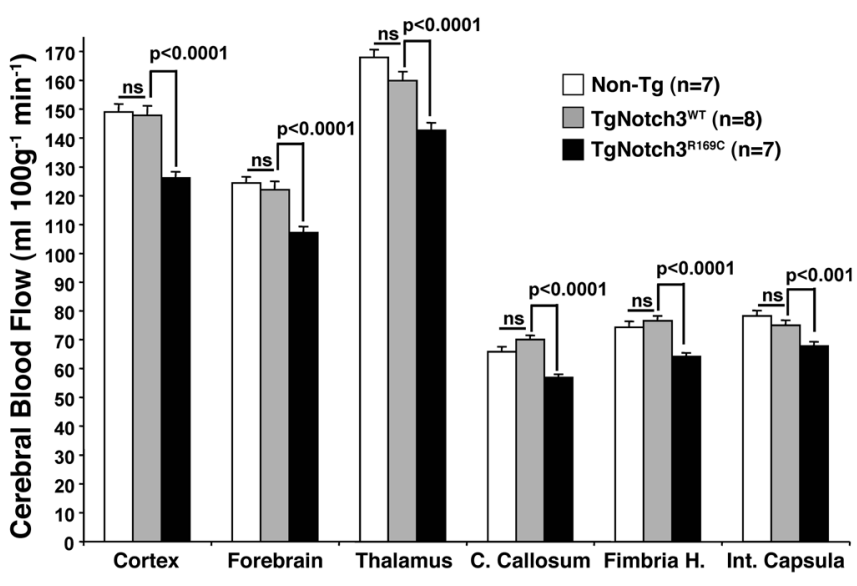

Figure 5

Decreased resting CBF in aged TgNotch $3^{\mathrm{R} 169 \mathrm{C}}$ mice. Quantitative measurement of CBF through the neocortex, forebrain (including the striatum, pallidum, and amygdala), thalamus, corpus callosum, fimbria of the hippocampus, and internal capsula in 18-month-old TgNotch3 ${ }^{\mathrm{R} 169 \mathrm{C}} \mathrm{com}$ pared with TgNotch $3^{\mathrm{WT}}$ and nontransgenic mice showed diffuse cerebral hypoperfusion in mutant mice. Each of these large areas included 8-10 gray matter regions or $4-5$ white matter regions.

and constrict when blood pressure increases, within the range of $60-150 \mathrm{mmHg}$, to maintain roughly stable cerebral perfusion. We found impaired adaptive responses of the cerebral circulation in the face of acute changes of arterial pressure in TgNotch $3^{\mathrm{R} 169 \mathrm{C}}$ mice. Particularly, the lower limit of cortical blood flow (CoBF) autoregulation (90\% of baseline) was shifted from $60 \mathrm{mmHg}$ in control TgNotch $3^{\mathrm{WT}}$ and nontransgenic mice to higher blood pressure $(80 \mathrm{mmHg})$ in TgNotch $3^{\mathrm{R} 169 \mathrm{C}}$ mice (Figure $\left.7 \mathrm{~A}\right)$. Conversely, acute hypertension induced by phenylephrine infusion was associated with less CoBF increase in TgNotch $3^{\mathrm{R} 169 \mathrm{C}}$ mice compared with control age-matched TgNotch $3^{\mathrm{WT}}$ mice (Figure $7 \mathrm{~B}$ ). These findings are consistent with altered myogenic responses or increased vascular resistances in mutant mice. Moreover, although the full autoregulatory range toward provoked hypertension was not explored, the data suggest a right shift of the pressure-flow curve in the mutant mice similar to what is seen in mice with chronic hypertension. However it is remarkable that TgNotch $3^{\mathrm{R} 169 \mathrm{C}}$ mice exhibited normal resting arterial blood pressure (Supplemental Table 2).

Functional hyperemia relates to the increase in blood flow induced by increased synaptic activity in a particular brain region. We studied the response in the somatosensory cortex elicited by whiskers stimulation. TgNotch $3^{\mathrm{R} 169 \mathrm{C}}$ mice showed altered CoBF responses to this physiological activation. The $35.5 \%-36.6 \% \mathrm{CoBF}$ increase in the somatosensory cortex elicited in TgNotch $3^{\mathrm{WT}}$ or nontransgenic mice was significantly attenuated by $31 \%-33 \%$ in TgNotch $3^{\mathrm{R} 169 \mathrm{C}}$ mice (Figure $7 \mathrm{C}$ ). In contrast, the increase in CoBF produced by hypercapnia was unaltered in mutant mice, indicating that the deficit of vasodilation was not due to a generalized vascular dysfunction at this age (Supplemental Figure 9).

To probe the vascular dysfunction caused by mutant Notch3, posterior cerebral arteries from mutant and control mice were dissected under a microscope, mounted on a pressure arteriograph, and stimulated with vasoactive drugs or pressure changes. The relaxation to sodium nitroprusside and the contractile response to phenylephrine of TgNotch $3^{\mathrm{R} 169 \mathrm{C}}$ arteries were comparable with those of TgNotch $3^{\mathrm{WT}}$ and nontransgenic littermate mice (data not shown). However, the pressure-induced contraction was markedly attenuated in TgNotch $3^{\mathrm{R} 169 \mathrm{C}}$ arteries compared with TgNotch3 ${ }^{\mathrm{WT}}$ and nontransgenic arteries (Figure 8A). For example, at $75 \mathrm{mmHg}$, myogenic tone was reduced by $26 \%-30 \%$ in $\operatorname{TgNotch} 3^{\mathrm{R} 169 \mathrm{C}}$ mice $(P<0.01)$. Moreover, passive internal diameter, which relates to maximal dilation of the artery after deactivation of smooth muscle cells with EGTA and sodium nitroprusside, was significantly less in cerebral arteries of TgNotch $3^{\mathrm{R} 169 \mathrm{C}}$ mice than in those of TgNotch $3^{\mathrm{WT}}$ and nontransgenic control mice at all pressures applied above $25 \mathrm{mmHg}$ (Figure 8B). Consequently, the "dilator reserve," defined as the difference between active and passive diameter, was strikingly reduced in TgNotch $3^{\mathrm{R} 169 \mathrm{C}}$ mice $\left(26.2 \pm 2.3 \mu\right.$ m versus $38.6 \pm 2.5 \mu \mathrm{min}$ TgNotch $3^{\mathrm{WT}}$ and $44.1 \pm 2.1 \mu \mathrm{m}$ in nontransgenic mice at $75 \mathrm{mmHg} ; P<0.01)$. Of note, wall thickness was not significantly different in cerebral arteries of TgNotch $3^{\mathrm{R} 169 \mathrm{C}}$ mice than in those of control TgNotch $3^{\mathrm{WT}}$ and nontransgenic mice (data not shown). Together, these data provide evidence that mutant Notch3 alters the mechanical properties and myogenic responses of cerebral arteries and that cerebrovascular dysfunction is an early event in the disease process.

\section{Discussion}

A CADASIL mouse model with brain lesions. Although NOTCH3 mutations that cause CADASIL were identified more than a decade ago, progress toward understanding the underlying mechanisms of brain injury has been hampered by the absence of an animal model exhibiting brain parenchyma lesions $(24,25)$. Here, we report the introduction of a CADASIL-causing Notch 3 mutation into a PAC and the development of a transgenic mouse model that shows an accurate cell type-specific expression of this large DNA construct that mimicked several of the fundamental pathological aspects of CADASIL. Specifically, mutant mice develop early onset pathognomonic Notch $3^{\mathrm{ECD}}$ aggregates and GOM deposits in the brain vessels, slowly progressive degeneration of the white matter, and cerebral hypoperfusion that parallel features of CADASIL patients in kind and progression. NOTCH $3^{\mathrm{ECD}}$ aggregates and GOM deposits have been reported in human CADASIL patients very early in the course of the disease, prior to the appearance of clinical symptoms and MRI changes (ref. 38 and our unpublished observations). Isolated white matter lesions in transgenic mutant mice correlate with human data, since isolated leukoaraiosis, the presumed MRI correlate of white matter damage in humans, occurs in human CADASIL patients many years before lacunar infarcts and clinical symptoms appear. Moreover, our finding of hypoperfusion in both normal-appearing neocortex and damaged white matter is consistent with human studies (33).

Notch 3 transgene expression. The main difference between this transgenic mouse model and prior mouse models, which did not develop brain damages (22-24), is the higher overexpression level of mutant Notch3. Accordingly, we found that the higher (line 88) but not the lower-expression mutant transgenic line 92 developed brain lesions (data not shown). However, whether stronger overexpression of mutant Notch3 may produce a more robust CADASIL mouse model is uncertain. Our data show that both wild-type and mutant PAC transgenic mice developed Notch3 cytoplasmic inclusions highly reminiscent of those seen in transfected cells overloaded with Notch proteins (ref. 30 and our unpublished observations). Accordingly, frequency of cytoplasmic inclusions was lower in line 92 than in lines 88 and 129 and comparable between lines 88 and 129 , which express a similar level of transgene, supporting the idea 
A
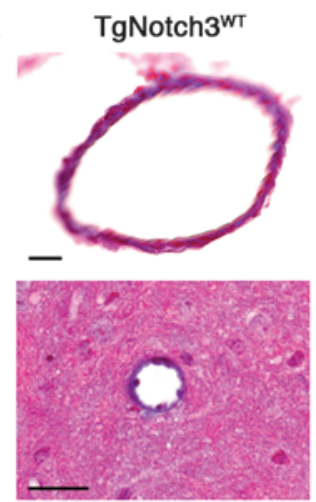

C

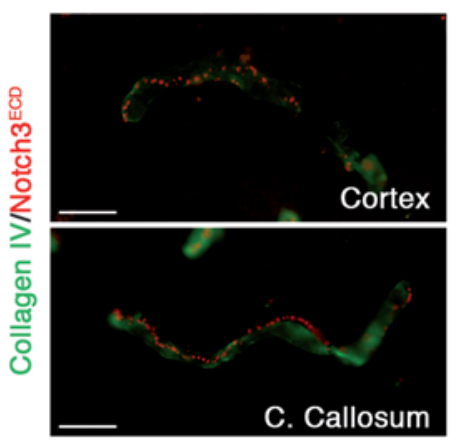

TgNotch3 ${ }^{\text {म169C }}$
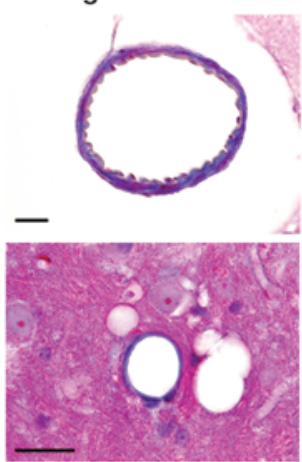

B

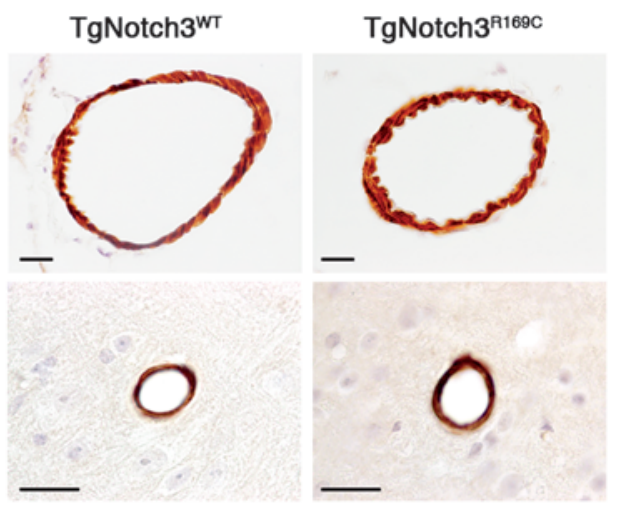

D ns

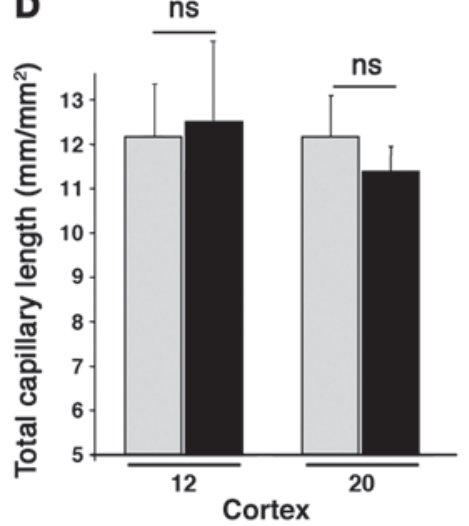

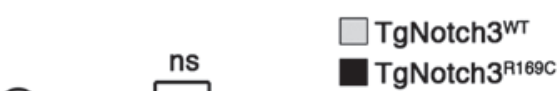

TgNotch3
TgNotch
R169c

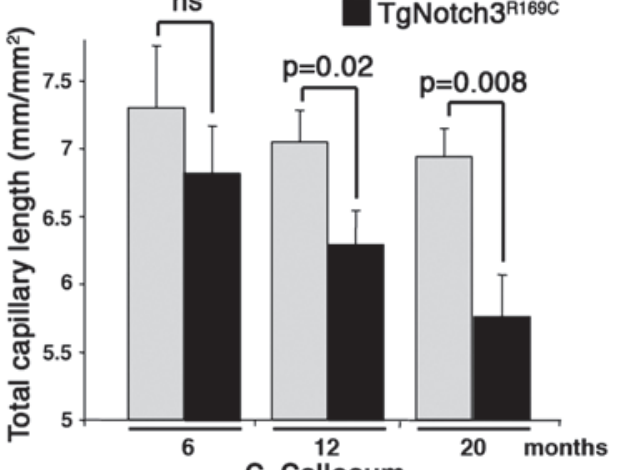

C. Callosum

\section{Figure 6}

Arterial structure is preserved, but capillary density is progressively reduced in the white matter of TgNotch3 ${ }^{\mathrm{R} 169 \mathrm{C}}$ mice. (A) Masson's trichrome staining showing intact pial artery (upper panels) and small artery (lower panels) of 20-month-old TgNotch3 ${ }^{\text {R169C }}$ mice. (B) Labeling for smooth muscle myosin heavy chain revealing a continuous rim of smooth muscle cells in pial and small artery of 20-month-old TgNotch3R169C mice. (C) Double labeling for Notch3ECD (5E1, red) and collagen IV (green) of capillaries in the cortex and the corpus callosum of 12-month-old TgNotch3 ${ }^{\mathrm{R} 169 \mathrm{C}}$ mice showed robust aggregation of Notch3ECD in both capillaries. (D) Mean total capillary length ( $\mathrm{mm}^{\mathrm{E}}$ of $\mathrm{CD} 31^{+} \mathrm{structures}$ per $\mathrm{mm}{ }^{2}$ ) in the cortex of 12- and 20-month-old TgNotch3 ${ }^{\mathrm{R} 169 \mathrm{C}}$ mice and the corpus callosum of 6-, 12-, and 20-month-old TgNotch3 ${ }^{\mathrm{R} 169 \mathrm{C}}$ mice compared with age-matched TgNotch $3^{\mathrm{WT}}$ mice showed progressive age-related reduction in capillary density in the white matter of mutant mice $(n=4-5$ mice per group). Scale bars: $25 \mu \mathrm{m}$.

that formation of these inclusions is related to the overexpression level of the transgene. Moreover, the finding that brain and vessels of wild-type PAC transgenic mice were histologically and functionally normal argues against a pathogenic role of these inclusions. Importantly, we found that the presence of Notch3 cytoplasmic inclusions and Notch ${ }^{\mathrm{ECD}}$ aggregates in mutant mice were mutually exclusive within a given cell and that occurrence of Notch $3^{\mathrm{ECD}}$ aggregates was delayed in capillaries, which exhibited a high frequency of inclusions, as compared with arteries that occasionally showed these inclusions. Together these findings lead us to propose that overexpressing Notch3 above a critical threshold may favor the sequestration of Notch3 precursor, shorten exposure to the pathogenic Notch3 polypeptides, and thus delay initiation of the disease process. Another important difference between the present and our prior transgenic mice is the distribution pattern of Notch 3 transgene. While brain arteries were targeted in both transgenic mice, capillaries were targeted in the former but not the latter because the SM22 $\alpha$ promoter element drives transgene expression in smooth muscle cells but not in pericytes (39). This may be particularly relevant with regard to our data supporting an involvement of the microcirculation in the mechanisms of brain lesions (see below).
White matter lesions and brain bypoperfusion. There is compelling evidence that chronic hypoperfusion is important in the pathogenesis of leukoaraiosis (40). CBF studies in human patients have documented hypoperfusion in the white matter within regions of leukoaraiosis as well as, to a lesser extent, in the normal-appearing white matter (41). Experimental studies in rodents have demonstrated that chronic brain hypoperfusion, induced by permanent ligation or stenosis of both common carotid arteries, produced lesions located predominantly in the white matter, with vacuolation, astrogliosis, and demyelination (2, 42, 43). Importantly, studies in the mouse in which the level of hypoperfusion was controlled by adjusting the internal diameter of microcoils placed around the carotid arteries provided evidence that the magnitude of hypoperfusion determined the ratio of white versus gray matter injury. Specifically, mild hypoperfusion caused selective damage of the white matter, while more severe hypoperfusion produced both white and gray matter lesions $(43,44)$. Herein, we show that aged mutant transgenic mice display moderate widespread reductions in resting $\mathrm{CBF}$, with a trend toward stronger $\mathrm{CBF}$ reduction in the white matter bundles. Of note, white matter hypoperfusion is presumably underestimated due to postmortem diffusion of 
A

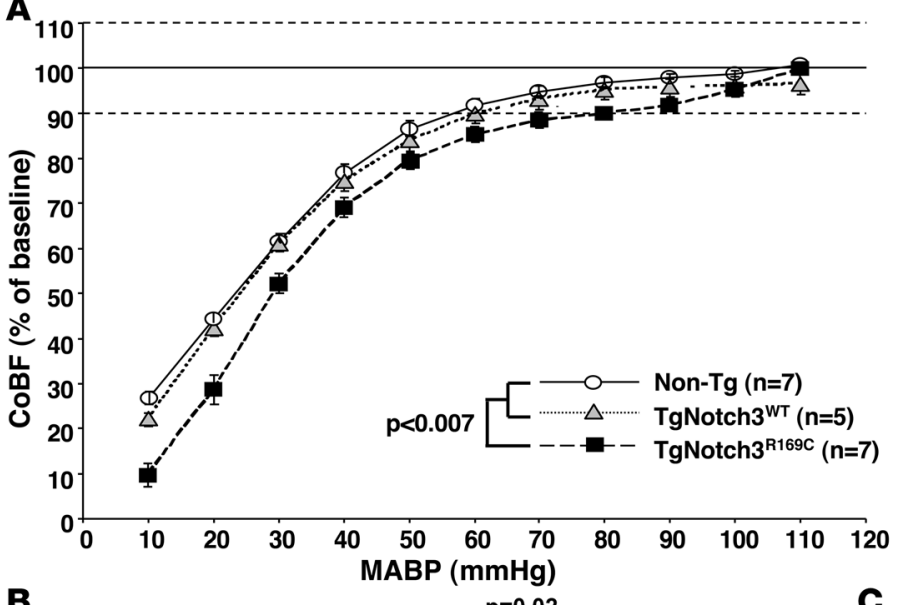

B
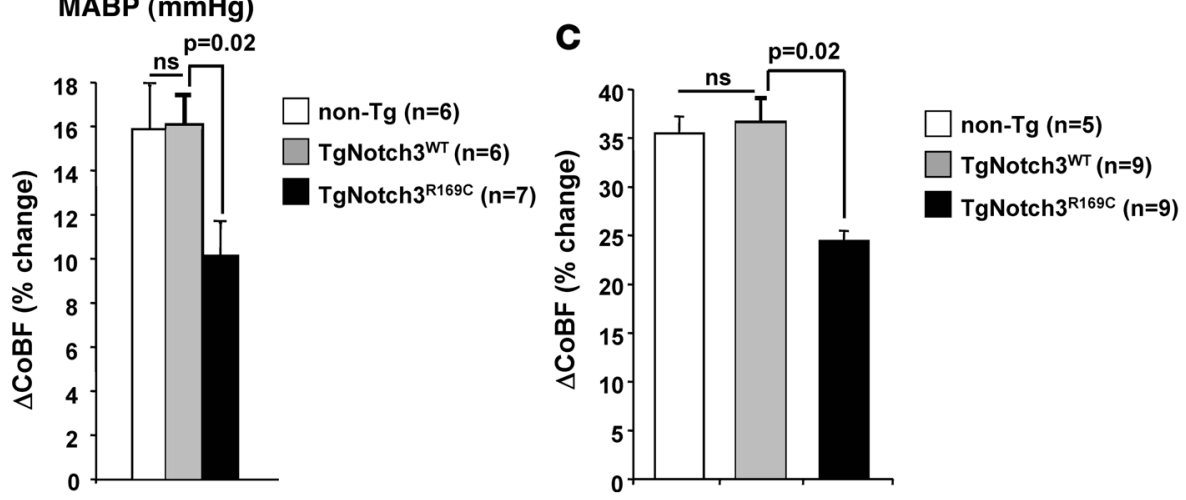

Figure 7

Impaired cerebrovascular autoregulation and attenuated functional hyperemia in young TgNotch3 ${ }^{\mathrm{R} 169 \mathrm{C}}$ mice. (A) Autoregulation curves of CoBF in 5-month-old nontransgenic, TgNotch ${ }^{\mathrm{WT}}$, and TgNotch3 ${ }^{\mathrm{R} 169 \mathrm{C}}$ mice in response to provoked hypotension showed that CoBF remained above $90 \%$ (lower dotted line, considered as the lower limit of autoregulation) up to $60 \mathrm{mmHg}$ in TgNotch3WT and nontransgenic mice, but only up to $80 \mathrm{mmHg}$ in TgNotch3 ${ }^{\mathrm{R} 169 \mathrm{C}}$ mice. (B) CoBF (in percent change) changes in response to arterial blood pressure increase ( $\triangle \mathrm{MABP}$, in mmHg) induced by phenylephrine infusion $(40 \mu \mathrm{g} / \mathrm{kg})$, in 5-month-old nontransgenic, TgNotch3 ${ }^{\mathrm{WT}}$, and TgNotch3 ${ }^{\mathrm{R} 169 \mathrm{C}}$ mice showing a smaller CoBF increase in mutant mice. (C) CoBF increase (\%) in the somatosensory cortex in response to whisker stimulation in 5- to 6-month-old nontransgenic, TgNotch ${ }^{\mathrm{WT}}$, and $\mathrm{TgNotch} 3^{\mathrm{R} 169 \mathrm{C}}$ mice showing attenuation of functional hyperemia in mutant mice.

the tracer from large gray matter regions of higher blood flow to the surrounded thin white matter bundles of half flow values (45). The 2 following observations support a causal role of hypoperfusion in the pathogenesis of white matter lesions in this mouse model rather than a secondary reduction of perfusion due to reduced demand of damaged tissue. First, at 20 months of age, CBF was decreased not only in the damaged white matter but also in undamaged regions such as the neocortex. Second, we found that $\mathrm{CBF}$ reduction was already detectable at 12 months of age before the appearance of brain lesions. Additional evidence linking white matter damages to hypoperfusion in mutant transgenic mice comes from the striking similarity between the lesions we observed here and those reported in these rodent models of chronic moderate hypoperfusion (43). Finally, our finding that overexpression of mutant Notch3 is predominant in vascular cells and negligible in other cell types except in Bergmann glial cells of the cerebellum further supports the notion that white matter lesions in this mouse model are caused by vascular insufficiency, rather than by primary defects in oligodendrocytes or other cell types of the white matter. Definite proof might require generation and analysis of mice expressing mutant Notch3 specifically in nonvascular cells.
Mechanisms of brain lesions and bypoperfusion. While a number of mechanisms have been proposed to account for brain parenchyma damages and hypoperfusion in ischemic cerebral SVD, including CADASIL, the earliest pathological events that initiate the lesions are not well understood. The present work provide compelling evidence implicating cerebrovascular dysfunction in addition to microcirculatory rarefaction as the earliest consequences of pathogenic mutant Notch3 expression. First, we identified in mutant mice impaired cerebrovascular autoregulation, which was apparent as early as 5-6 months of age and thus several months before the first histological alterations in white matter. This result is consistent with our previous work with transgenic mice expressing the R90C NOTCH3 mutant (46). Second, we document that young TgNotch $3^{\mathrm{R} 169 \mathrm{C}}$ mice have attenuated functional hyperemia. Third, our experiments on isolated arteries demonstrate that mutant Notch3 affects at an early age (a) the functional properties of cerebral arteries, with an attenuation of the myogenic responses, and (b) the mechanical properties of these arteries, with a reduction in vessel diameter. Both alterations contribute to a decrease in the dilator reserve. Additionally, according to Poiseuille's law, where the resistance of an arterial segment is inversely proportional to the fourth power of the vessel radius, the decrease in arterial caliber will result in markedly increased vascular 

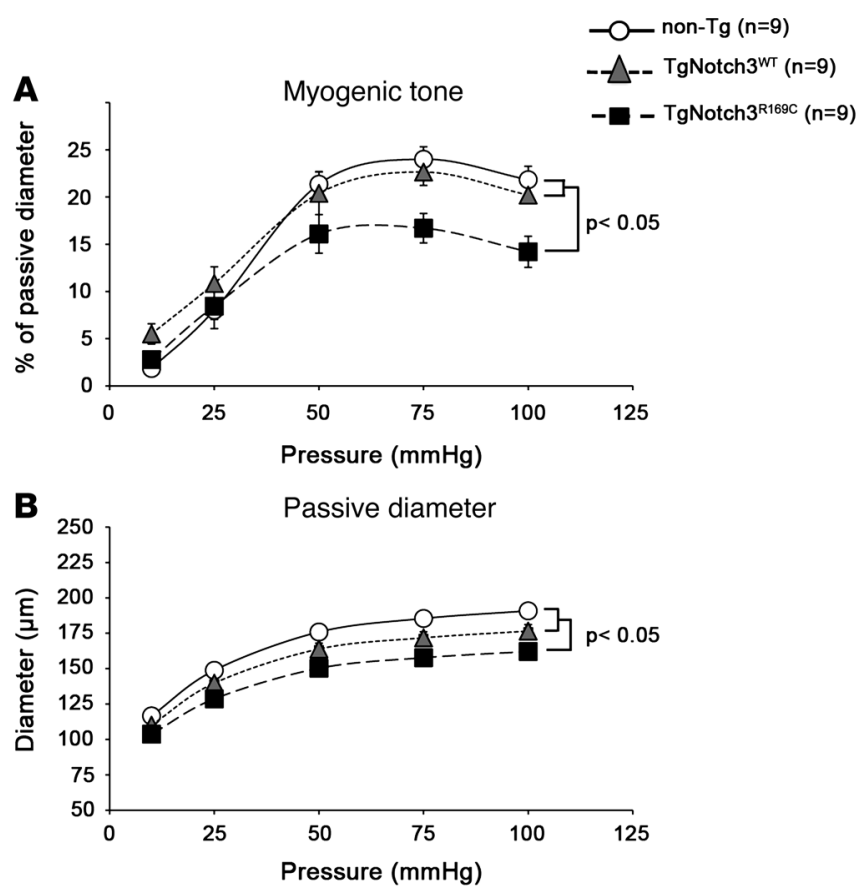

resistance and decreased blood flow. Fourth, we identified a progressive rarefaction of brain capillaries in the white matter and provided compelling evidence that it is likewise an early manifestation. Particularly, we show that capillary reduction in the white matter occurs prior to $\mathrm{CBF}$ reduction in this territory, arguing against a secondary reduction by pruning due to reduced blood flow. Instead, this observation favors the opposite causal sequence that capillary rarefaction contributes to $\mathrm{CBF}$ reduction. Our overall findings thus support the hypothesis that mechanical and functional alterations of cerebral arteries act in concert with reduction of the capillary bed to reduce baseline cerebral perfusion and damage white matter, which is highly vulnerable to a fall in perfusion. Moreover, alteration of cerebrovascular autoregulation is anticipated to exacerbate reductions in $\mathrm{CBF}$ when arterial blood pressure drops, particularly in the white matter, which is supplied by terminal vessels with limited collateral flow (37). Importantly, we demonstrate that neuropathological changes in this mouse model occur in the absence of histologically detectable alterations of the structure of brain arteries. Thus, contrary to the common belief, our data indicate that smooth muscle cell loss and fibrosis of the arterial wall do not contribute to disease initiation, but this is not to say that, once present, these structural lesions may not aggravate cerebrovascular dysfunction and exacerbate brain lesions already in progress.

Why capillary density is predominantly reduced in the white matter is unclear at the moment, since it contrasts with a broader expression of mutant Notch3 and deposition of Notch $3^{\mathrm{ECD}}$ aggregates along the brain capillary bed. Future studies should aim at identifying the mechanisms of capillary reduction.

It is worth mentioning that TgNotch $3^{\mathrm{R} 169 \mathrm{C}}$ mice do not fully recapitulate the CADASIL disease, as they do not develop lacunar infarcts. This may be accounted for by differences between humans and mice regarding brain size, angioarchitecture, as well as lower white matter/gray matter ratio in the mouse. As such, brain regions that are prominently vulnerable to a fall in perfusion may be more discrete in mice than in humans.

\section{Figure 8}

Altered mechanical properties and myogenic responses in young TgNotch3 ${ }^{\text {R169C }}$ mice. (A) Response of cerebral artery to stepwise increase in pressure (myogenic tone) in 6-month-old nontransgenic, TgNotch3 ${ }^{\mathrm{WT}}$, and TgNotch3 ${ }^{\mathrm{R} 169 \mathrm{C}}$ mice showed that myogenic tone was significantly attenuated in TgNotch $3^{\mathrm{R} 169 \mathrm{C}}$ mice. (B) Internal diameter/ pressure relationships in cerebral arteries during maximal dilation with EGTA and sodium nitroprusside (passive diameter) in 6-month-old nontransgenic, TgNotch $3^{W T}$, and TgNotch $3^{R} 169 \mathrm{C}$ mice. Passive diameters were significantly less in TgNotch $3^{\mathrm{R} 169 \mathrm{C}}$ mice compared with both nontransgenic and TgNotch3 ${ }^{W T}$ mice.

In summary, our data show that cardinal pathological features of CADASIL can be recapitulated in the mouse when overexpressing an appropriate amount of mutant Notch3 in an accurate spatiotemporal pattern. Our findings provide compelling evidence for cerebrovascular dysfunction and microcirculatory failure as the earliest consequences of pathogenic mutant Notch3, culminating in progressive hypoperfusion and white matter damage in this novel genetic model of ischemic cerebral SVD. These mice will be valuable for exploring the mechanisms and the efficacy of therapeutic interventions for CADASIL and also nonhereditary ischemic SVD.

\section{Methods}

\section{Generation of transgenic mice}

The 172-kb PAC clone RPCI31.78K09 containing the rat genomic Notch3 locus was identified by PCR pool screening of the rat PAC library (27). We manipulated the Notch3 PAC clone in E. coli using a temperature-sensitive shuttle vector-based system for homologous recombination as described by Yang et al. (28), an approach that left only the desired mutation, to create the R169C CADASIL amino acid substitution. Fidelity of the final product was determined by restriction enzyme fragment mapping and pulsed field gel electrophoresis analyses, PCR and DNA sequencing. PAC DNA was purified and prepared for microinjection using the standard protocols (47, 48). AscI linearized Notch $3^{\mathrm{WT}}$ and Notch $3^{\mathrm{R} 169 \mathrm{C}} 170-\mathrm{kb}$ PAC constructs were injected into FVB/N mouse oocytes. Positive transgenic founder mice were identified for each construct as follows: (a) one FVB/N-TgNotch3 (line 129)/ $\mathrm{Bbb}$ mouse expressing the rat wild-type (not modified) Notch3-containing 170-kb PAC clone and (b) two mice FVB/N-TgNotch3 (line 88)/Bbb and FVB/N-TgNotch3 (line 92)/Bbb, expressing the CADASIL-causing R169C Notch3. Strains are referred to as TgNotch $3^{\mathrm{WT}}$ (line 129), TgNotch $3^{\mathrm{R} 169 \mathrm{C}}$ (line 88), and TgNotch $3^{\mathrm{R} 169 \mathrm{C}}$ (line 92) throughout the manuscript. Integrity of full-length genomic integration of the $170-\mathrm{kb}$ mutant and wild-type Notch3 PACs in all 3 established lines (lines 88, 92, and 129) was assessed by Southern blotting and PCR analysis. Mice were backcrossed a minimum of 10 times onto an FVB/N background and maintained in this strain. All transgenic mice analyzed carried the transgene at the heterozygous state. All experiments described in this study were conducted in full accordance with the guidelines of the Institutional Animal Care and Use Committee of the Max-Delbruck-Center for Molecular Medicine (Landesamt für Gesundheit und Soziales G0034/98) and INSERM (Paris, France; Ile de France committee 4), with every effort made to minimize the number of animals used.

\section{Northern blot and RT-PCR}

Total RNA was extracted from the brain using TRIzol reagent (Invitrogen). Twenty micrograms of RNA were electrophoresed on a $6.8 \%$ formaldehyde, $1 \%$ agarose gel, transferred to Hybond $\mathrm{N}+$ nylon membrane (Amersham Biosciences), and probed with a 5 ' rat Notch 3 cDNA fragment (gi: 9966774; nt 1199-2005). 
We reverse transcribed $3 \mu \mathrm{g}$ of DNaseI-treated RNA into cDNA using the M-MLV reverse transcriptase (Invitrogen). Brain cDNA was prepared from 3 different animals of each genotype, and each sample was assayed in duplicate. Primers for RT-PCR were designed to recognize both endogenous mouse Notch3 and exogenous rat Notch3 (5'-GCAGGTGATGGCCTAAGTTC-3', 5'-GGTCAGCCCCTACCCATTAT-3').

\section{Western blot}

Brain samples were homogenized in ice-cold RIPA buffer $(150 \mathrm{mM} \mathrm{NaCl}$, $50 \mathrm{mM}$ Tris-HCL, $1 \%$ NP-40, $0.1 \%$ SDS, $0.5 \%$ sodium desoxycholate) supplemented with protease inhibitors $(1 \times$ complete protease inhibitor mixture; Roche Diagnostics). Protein extract $(40 \mu \mathrm{g})$ of each sample was separated in $6 \%$ SDS-PAGE, transferred to nitrocellulose membrane, incubated with mouse monoclonal anti-Notch3 ${ }^{\mathrm{ECD}}$ antibody (12) (clone 5E1, 1: 500 dilution), and followed by peroxidase-conjugated goat anti-mouse. Mouse anti- $\alpha$-tubulin (clone DM 1A, 1:15,000 dilution) (Sigma-Aldrich) and rabbit anti-smooth muscle myosin heavy chain (1:5,000 dilution) (Biomedical Technologies Inc.) were used to check that equal amounts of protein extracts were loaded.

\section{In situ bybridization}

We used an 807-bp PCR product of rat Notch3 mRNA (gi: 9966774; nt 1199-2005) cloned into pCR-BluntII-TOPO vector (Invitrogen) as a template to synthesize antisense cRNA probes. The plasmid was linearized with Sac-I and transcribed with T7 RNA polymerase in the presence of $\left[{ }^{35} \mathrm{~S}\right]$-UTP. In situ hybridization was performed as described in Supplemental Methods. Sections were examined with dark field and bright field illumination on a Leica DMR microscope.

\section{Histology, immunohistochemistry, and GOM deposit analysis}

Histopathological features of the transgenic mice were studied by analyzing 1-, 6-, 12- and 20-month-old nontransgenic and transgenic mice. Tissues were processed for histology, immunohistochemistry, and electron microscopy analysis as described in the Supplemental Methods.

\section{Quantification of vacuoles and GFAP area}

Sagittal sections stained with hematoxylin and eosin $(n=9$ sections per mouse per region) or GFAP ( $n=8-22$ sections per mouse per region) taken lateral -0.5 to $-2.0 \mathrm{~mm}$ to the midline were selected according to the mouse brain atlas (49). Images that covered most to all the regions of interest were collected from a CCD color video camera and analyzed using software NIS-Element BR 3.0 (Nikon). Quantitative comparisons were performed on sections from mutant and wild-type animals processed in parallel, with digital images obtained using the same settings simultaneously per group age. The area of vacuoles was expressed as a percentage of the total surface for each region, and vascular area in each region of interest was subtracted manually from vacant area. GFAP-immunopositive area was expressed as a percentage of the total image area.

\section{Capillary length measurement}

Mice were deeply anesthetized with sodium pentobarbital and perfused transcardially with $50 \mathrm{ml}$ of $1 \times$ PBS, followed by $200 \mathrm{ml}$ of $4 \%$ paraformaldehyde in phosphate buffer. Brain was harvested, half cut, postfixed for 24 hours in $4 \%$ paraformaldehyde at $4^{\circ} \mathrm{C}$, and cryoprotected in $15 \%-30 \%$ sucrose at $4^{\circ} \mathrm{C}$ and embedded in OCT (Tissue-Tek). Freefloating saggital sections ( $20 \mu \mathrm{m} ; 6$ sections per mouse) were blocked by $5 \%$ goat serum and $0.4 \%$ Triton $\mathrm{X}-100$ for 2 hours at room temperature and incubated with rat monoclonal anti-mouse CD31 (1:50; BD Biosciences - Pharmingen) in $5 \%$ goat serum and $0.4 \%$ Triton X-100 overnight at $4{ }^{\circ} \mathrm{C}$. Sections were then washed and incubated with Alexa Fluor 594 anti-rat IgG (1:500; Invitrogen) for 2 hours at room temperature. Images were taken using a Nikon eclipse $80 \mathrm{i}$ fluorescent microscope. Images $(\times 40$ objective lens) that covered most to all the region of study were collected from a CCD color video camera and analyzed using software NIS-Element BR 3.0 (Nikon). The total length of CD31+ blood vessels was expressed as the total length $(\mathrm{mm})$ of $\mathrm{CD} 31^{+}$vessels per unit area $\left(\mathrm{mm}^{2}\right)$. Sections from mutant and wild-type animals were processed in parallel.

\section{$B B B$ analysis}

Electron microscopy. Mice were deeply anesthetized with sodium pentobarbital and perfused transcardially with $20 \mathrm{ml}$ of $0.5 \%$ paraformaldehyde and $0.05 \%$ glutaraldehyde and then with $500 \mathrm{ml}$ of $2 \%$ paraformaldehyde and $2 \%$ glutaraldehyde in phosphate buffer. Brain samples $(0.5-1 \mathrm{~mm})$ were dissected under microscope within regions of interest and postfixed for 12 hours at $4^{\circ} \mathrm{C}$. Samples were further postfixed in $1 \%$ osmium tetroxide for 2 hours at $4^{\circ} \mathrm{C}$, dehydrated with graded concentrations of alcohol, and embedded in Epon. Ultrathin sections were processed as described above and observed with a Philips CM100 electron microscope.

Biotinylated 70-kDa dextran fluorescence profiling. Mice were injected via the tail vein with $2 \mathrm{mg}$ of biotinylated 70-kDa dextran (Sigma-Aldrich), dextran was allowed to circulate for 2 hours, and mice were sacrificed by lethal anesthesia using halothane and decapitation. Brain was postfixed for 24 hours in $4 \%$ paraformaldehyde at $4{ }^{\circ} \mathrm{C}$ and then incubated in 15\%-30\% sucrose for cryoprotection. Free-floating sections $(20 \mu \mathrm{m})$ were further processed for FITCstreptavidin and CD31 co-immunostaining as described above. Mutant and wild-type animals were processed in parallel. Dextran and CD31 fluorescence was visualized with a laser-scanning confocal imaging system (Leica TCS SP5 AOBS tandem resonant scanner). Optical slices were processed by LAS AF Lite imaging software (Leica), and reconstructed images were evaluated for dextran present outside the CD31-delineated intraluminal space.

\section{CBF analysis}

Autoradiographic measurement of resting $C B F$. CBF was measured by the quantitative autoradiographic technique in the conscious mouse, using $\left[{ }^{14} \mathrm{C}\right]$ iodoantipyrine (IAP) as a diffusible tracer, following the protocol adapted for the mouse $(50,51)$. Under isoflurane anesthesia (2\% progressively reduced to $1.7 \%$ inhaled via a face mask in $25 \% \mathrm{O}_{2}$ and $75 \% \mathrm{~N}_{2}$ ), the femoral vessels were catheterized for tracer infusion, continuous arterial pressure monitoring, and collection of arterial blood samples. Tygon catheters were heparinized so as to provide $1,000 \mathrm{IU} / \mathrm{kg}$. Skin incisions were carefully sutured and covered with $2 \%$ lidocaine gel. Mice were held under minimal restraint and allowed to recover from anesthesia for 2 hours. Heart rate and arterial blood pressure were continuously monitored (PowerLab/8SP). Body temperature was monitored with a rectal probe and maintained between $37.0^{\circ} \mathrm{C}$ and $37.7^{\circ} \mathrm{C}$. Arterial blood gases, $\mathrm{pH}$, and hematocrit were measured shortly before CBF measurement (Ciba-Corning 248). IAP (ARC, $54 \mathrm{mCi} / \mathrm{mmol}$ in saline, $100 \mu \mathrm{Ci} / \mathrm{kg}$ in $0.2 \mathrm{ml}$ ) was infused intravenously at a constant rate using a programmable infusion pump (Harvard 44) to produce a ramp arterial concentration curve of the tracer. Simultaneously, a series of $8-10$ arterial blood samples were serially collected during 30 seconds. To avoid hypotension, small sample volumes (10-20 $\mu \mathrm{l})$ were taken and a similar volume of tracer solution was simultaneously infused. At the end of the infusion, mice were decapitated, and their whole heads were rapidly frozen in isopentane chilled at $-30^{\circ} \mathrm{C}$. Sagittal brain sections $(20 \mu \mathrm{m})$ were cut on a cryostat, mounted on glass slides, and either used for autoradiography or stained with cresyl violet for histological examination. The $\left[{ }^{14} \mathrm{C}\right]$-tracer concentration in brain was assessed by densitometric analysis of autoradiograms (MCID; Imaging Research) using calibrated ${ }^{14} \mathrm{C}$ standards on the film. Regional CBF ( $\mathrm{ml} / 100 \mathrm{~g}$ of brain tissue/min) was calculated using the local tracer concentration accumulated in brain tis- 
sue and the time course of arterial tracer concentration counted by liquid scintillation in arterial plasma samples. We also applied the washout coefficient correction introduced by Jay and colleagues in blood flow calculation to improve accuracy in low-bloodflow values (50). We identified 25 white and 58 gray matter regions of interest on the cresyl violet-stained sections using the mouse brain atlas (49). Regions were pooled in large regional areas for statistical analysis.

Determination of CoBF by laser Doppler flowmetry. Surgical preparation was carried out under isoflurane anesthesia as described above. The head was then placed in a Kopf stereotaxic frame, and the left and right parietal bones $\left(\sim 2.5-\mathrm{mm}^{2}\right.$ area $)$ were thinned to translucency with a dental drill. Two laser Doppler flowmeter probes (Moor Instrument MBF3-Dual) were then carefully positioned over the cortex to avoid large vessels. Heart rate, blood pressure, and CoBF were continuously monitored (PowerLab/8SP). Zero values for CoBF were obtained at the end of experiment after circulatory arrest by an overdose of pentobarbital. Cerebrovascular autoregulation and response to hypercapnia were measured in conscious animals as described earlier (46) and in Supplemental Methods. Responses to whisker stimulation were measured on isoflurane-anesthetized animals. Laser Doppler flowmeter probes were stereotaxically placed above the right and left somatosensory area of the barrel field (S1bf) at the following coordinates bregma 0.0 to -1.2 , lateral 2.8 to $3.5 \mathrm{~mm}$. After surgical preparation, isoflurane was reduced to $1.2 \%-1.5 \%$, and measurements started when responsiveness to hypercapnia had recovered. Vibrissae were clipped at $5 \mathrm{~mm}$ and gently manually stimulated for 30 seconds with a cotton-tipped applicator, alternatively on the right and left whiskers. Unilateral whisker stimulation produced increases in $\mathrm{CoBF}$ in the contralateral somatosensory cortex that reached a plateau. $\mathrm{CoBF}$ responses were expressed as the percentage increase over the baseline before stimulation. Four responses were retained for each mouse. Responses associated with bilateral CoBF changes or changes in arterial blood pressure or heart rate were discarded.

\section{Vascular function analysis}

The first segment of the posterior cerebral artery was chosen for this analysis because it exhibits a lower number of collateral branches as compared with other brain arteries. A 3- to 5-mm-long arterial segment was dissected under a light microscope and mounted in a video-monitored perfusion system (Living Systems Instrumentation) as previously described (19). Briefly, arterial segment was cannulated on 2 glass micropipettes, using nylon monofilaments to secure both ends and close collateral branches when present, and bathed in a $5-\mathrm{ml}$ chamber containing a physiological salt solution maintained at a $\mathrm{pH}$ of $7.4, \mathrm{pO}_{2}$ of $160 \mathrm{mmHg}$, and a $\mathrm{pCO}_{2}$ of $37 \mathrm{mmHg}$. Pressure was controlled by a servo-perfusion system. Using a microscope and a video camera, vessel images were projected on a video monitor and internal lumen diameter was measured using an electronic dimension analyzer. Once prepared, arteries were allowed to stabilize at least 20 minutes at a pressure of $50 \mathrm{mmHg}$ before initiating analyses to acquire basal tone. Artery viability was tested using a potassium-rich solution $(80 \mathrm{mmol} / \mathrm{l})$ and phenylephrine $(10 \mu \mathrm{mol} / \mathrm{l})$. Myogenic tone was determined by increasing intraluminal pressure by steps from 10 to $100 \mathrm{mmHg}$. Diameters measured in physiological salt solution were considered as active diameter. Vasoreactivity was examined on vessels pressurized to $50 \mathrm{mmHg}$; contractile responses were assessed with phenylephrine $\left(10^{-9}\right.$ to $\left.10^{-4} \mathrm{~mol} / \mathrm{l}\right)$, and vasodilatory responses were tested with sodium nitroprusside $\left(10^{-10}\right.$ to $\left.10^{-4} \mathrm{~mol} / \mathrm{l}\right)$ on vessels preconstricted by $30 \%-35 \%$ with phenylephrine $(10 \mu \mathrm{mol} / \mathrm{l})$. After development of a stable baseline diameter, cumulative dose-response curves were obtained. At the end of each experiment, arteries were superfused with a $\mathrm{Ca}^{2+}$-free physiological salt solution containing EGTA ( $2 \mathrm{mmol} / \mathrm{l})$ and sodium nitroprusside $(10 \mu \mathrm{mol} / \mathrm{l})$. Pressure steps were repeated to determine the passive diameter of the arteries. After completion of pressure steps, maximally dilated vessels were fixed by suffusion in CARSON fixative solution, while intravascular pressure was maintained at $50 \mathrm{mmHg}$. Fixed vessels were processed, embedded in epon, and sectioned at $1 \mu \mathrm{m}$.

Pressure and diameter measurements were collected by a Biopac data acquisition system (Biopac MP 150) and continuously recorded. Data were analyzed with the Acqknowledge software. Results are given in micrometers for artery diameters. Myogenic tone was expressed as the percentage of passive diameter ([passive diameter - active diameter] $/$ passive diameter $\times 100$ ). Relaxation to sodium nitroprusside was expressed as the percentage of dilation of phenylephrine-induced preconstriction. Histological images of arteries were collected from a CCD color video camera, and wall thickness was determined using software NIS-Element BR 3.0 (Nikon).

\section{Statistics}

Data are expressed as mean \pm SEM. Two-group comparisons were analyzed using the 2-tailed $t$ test for independent samples. Multiple comparisons were evaluated by 1 -way ANOVA followed by Tukey's post-hoc test (Statview). Repeated-measures ANOVA (Statview) were used to compare curves of CoBF autoregulation, cumulative dose response to phenylephrine and sodium nitroprusside, myogenic tone, and passive diameters. CoBF changes were analyzed using a 2-way ANOVA for mixed model (fixed factor: line; random factor: mice) to take into account that several measurements came from the same animal. Calculations were made using PROC MIXED from SAS Software version 9.2. Normality of distribution was tested using the Shapiro-Wilk's test. In case of non-Gaussian distribution, logarithmic transformation was applied before ANOVA. Two-by-two comparisons were made using appropriate contrast and Tukey's correction for multiplicity. $P$ values less than 0.05 were considered significant.

\section{Acknowledgments}

This work was supported by grants from Institut de France (NRJ Foundation), the French National Research Agency (ANR-05MRAR no. A05206HS), and the NIH-National Neurological Disorders and Stroke Institute (R01 NS 054122) to A. Joutel and the German Ministry for Science and Education (BMBF) to N. Hubner. We thank Eric Vicault for assistance in conducting statistical analyses, the Institut Jacques Monod Bio-Imaging Core for confocal analysis, the Electron Microscopy Core at Institut du Fer-à-Moulin, and TAAM-Orleans (Karine Jambou) for animal housing. A. Joutel is partially supported by a Contrat d'interface INSERM-APHP.

Received for publication May 4, 2009, and accepted in revised form November 18, 2009.

Address correspondence to: Anne Joutel, Faculté de Médecine Paris7, site Villemin, 10 av de Verdun, 75010 Paris, France. Phone: 331-5727-8593; Fax: 331-5727-8594; E-mail: anne.joutel@univparis-diderot.fr. Or to: Norbert Hubner, MDC, Robert-Rossle-Str. 10, 13125 Berlin, Germany. Phone: 4930-9406-3512; Fax: 49309406-3147; E-mail: nhuebner@mdc-berlin.de.
1. Ringelstein EB, Nabavi DG. Cerebral small vessel diseases: cerebral microangiopathies. Curr Opin

Neurol. 2005;18(2):179-188.

2. Hainsworth AH, Markus HS. Do in vivo experi- mental models reflect human cerebral small vessel disease? A systematic review. J Cereb Blood Flow Metab. 2008;28(12):1877-1891.

3. Joutel A, et al. Notch3 mutations in CADASIL, a hereditary adult-onset condition causing stroke and dementia. Nature. 1996;383(6602):707-710.

4. Dichgans M. Genetics of ischaemic stroke. Lancet Neurol. 2007;6(2):149-161. 
5. Chabriat H, Joutel A, Dichgans M, TournierLasserve E, Bousser MG. Cadasil. Lancet Neurol. 2009;8(7):643-653.

6. Dichgans $M$, et al. The phenotypic spectrum of CADASIL: clinical findings in 102 cases. Ann Neurol. 1998;44(5):731-739.

7. Opherk C, Peters N, Herzog J, Luedtke R, Dichgans $M$. Long-term prognosis and causes of death in CADASIL: a retrospective study in 411 patients. Brain. 2004;127(Pt 11):2533-2539.

8. Chabriat H, et al. Patterns of MRI lesions in CADASIL. Neurology. 1998;51(2):452-457.

9. Ruchoux MM, Guerouaou D, Vandenhaute B, Pruvo JP, Vermersch P, Leys D. Systemic vascular smooth muscle cell impairment in cerebral autosomal dominant arteriopathy with subcortical infarcts and leukoencephalopathy. Acta Neuropathol. 1995;89(6):500-512.

10. Kalimo H, et al. CADASIL: hereditary disease of arteries causing brain infarcts and dementia. Neuropathol Appl Neurobiol. 1999;25(4):257-265.

11. Miao Q, et al. Fibrosis and stenosis of the long penetrating cerebral arteries: the cause of the white matter pathology in cerebral autosomal dominant arteriopathy with subcortical infarcts and leukoencephalopathy. Brain Pathol. 2004;14(4):358-364.

12. Joutel A, et al. The ectodomain of the Notch 3 receptor accumulates within the cerebrovasculature of CADASIL patients. J Clin Invest. 2000;105(5):597-605.

13. Joutel A, et al. Strong clustering and stereotyped nature of Notch 3 mutations in CADASIL patients. Lancet. 1997;350(9090):1511-1515.

14. Peters N, Opherk C, Bergmann T, Castro M, Herzog J, Dichgans M. Spectrum of mutations in biopsy-proven CADASIL: implications for diagnostic strategies. Arch Neurol. 2005;62(7):1091-1094.

15. Tikka S, et al. Congruence between NOTCH3 mutations and GOM in 131 CADASIL patients. Brain. 2009;132(Pt 4):933-939.

16. Joutel A, et al. Skin biopsy immunostaining with a Notch3 monoclonal antibody for CADASIL diagnosis. Lancet. 2001;358(9298):2049-2051.

17. Ishiko A, et al. Notch 3 ectodomain is a major component of granular osmiophilic material (GOM) in CADASIL. Acta Neuropathol. 2006;112(3):333-339.

18. Domenga $V$, et al. Notch3 is required for arterial identity and maturation of vascular smooth muscle cells. Genes Dev. 2004;18(22):2730-2735.

19. Belin de Chantemele EJ, et al. Notch3 is a major regulator of vascular tone in cerebral and tail resistance arteries. Arterioscler Thromb Vasc Biol. 2008;28(12):2216-2224.

20. Peters N, Opherk C, Zacherle S, Capell A, Gempel P, Dichgans M. CADASIL-associated Notch3 mutations have differential effects both on ligand binding and ligand-induced Notch3 receptor signaling through RBP-Jk. Exp Cell Res. 2004;299(2):454-464.

21. Joutel A, Monet M, Domenga V, Riant F, TournierLasserve E. Pathogenic mutations associated with cerebral autosomal dominant arteriopathy with subcortical infarcts and leukoencephalopathy differently affect Jagged 1 binding and Notch3 activity via the RBP/JK signaling Pathway. Am J Hum Genet. 2004;74(2):338-347.

22. Monet M, et al. The archetypal R90C CADASILNOTCH3 mutation retains NOTCH3 function in vivo. Hum Mol Genet. 2007;16(8):982-992.

23. Monet-Lepretre M, et al. Distinct phenotypic and functional features of CADASIL mutations in the Notch3 ligand binding domain. Brain. 2009;132(Pt 6):1601-1612.

24. Lundkvist J, et al. Mice carrying a R142C Notch 3 knock-in mutation do not develop a CADASIL-like phenotype. Genesis. 2005;41(1):13-22.

25. Ruchoux MM, et al. Transgenic mice expressing mutant Notch3 develop vascular alterations characteristic of cerebral autosomal dominant arteriopathy with subcortical infarcts and leukoencephalopathy. Am J Pathol. 2003;162(1):329-342.

26. Dillon N, Grosveld F. Transcriptional regulation of multigene loci: multilevel control. Trends Genet. 1993;9(4):134-137.

27. Woon PY, et al. Construction and characterization of a 10-fold genome equivalent rat P1-derived artificial chromosome library. Genomics. 1998;50(3):306-316.

28. Yang XW, Model P, Heintz N. Homologous recombination based modification in Escherichia coli and germline transmission in transgenic mice of a bacterial artificial chromosome. Nat Biotechnol. 1997;15(9):859-865.

29. Eiraku M, et al. DNER acts as a neuron-specific Notch ligand during Bergmann glial development. Nat Neurosci. 2005;8(7):873-880.

30. Karlstrom H, Beatus P, Dannaeus K, Chapman G, Lendahl U, Lundkvist J. A CADASIL-mutated Notch 3 receptor exhibits impaired intracellular trafficking and maturation but normal ligandinduced signaling. Proc Natl Acad Sci U S A. 2002;99(26):17119-17124.

31. Tuominen S, et al. Positron emission tomography examination of cerebral blood flow and glucose metabolism in young CADASIL patients. Stroke. 2004;35(5):1063-1067.

32. Mellies JK, et al. SPECT study of a German CADASIL family: a phenotype with migraine and progressive dementia only. Neurology. 1998;50(6):1715-1721.

33. Chabriat $\mathrm{H}$, et al. Cerebral hemodynamics in CADASIL before and after acetazolamide challenge assessed with MRI bolus tracking. Stroke. 2000;31(8):1904-1912.

34. Jellinger KA. The enigma of vascular cognitive disorder and vascular dementia. Acta Neuropathol. 2007;113(4):349-388.

35. Pantoni L, Garcia JH. Pathogenesis of leukoaraiosis: a review. Stroke. 1997;28(3):652-659.

36. Zlokovic BV. The blood-brain barrier in health and chronic neurodegenerative disorders. Neuron. 2008;57(2):178-201.

37. Iadecola C. Neurovascular regulation in the normal brain and in Alzheimer's disease. Nat Rev Neurosci.
2004;5(5):347-360

38. Ebke $M$, et al. CADASIL: skin biopsy allows diagnosis in early stages. Acta Neurol Scand. 1997;95(6):351-357.

39. Moessler H, Mericskay M, Li Z, Nagl S, Paulin D, Small JV. The SM 22 promoter directs tissue-specific expression in arterial but not in venous or visceral smooth muscle cells in transgenic mice. Development. 1996;122(8):2415-2425.

40. Markus HS. Genes, endothelial function and cerebral small vessel disease in man. Exp Physiol. 2008;93(1):121-127.

41. O'Sullivan M, et al. Patterns of cerebral blood flow reduction in patients with ischemic leukoaraiosis. Neurology. 2002;59(3):321-326.

42. Otori T, Katsumata T, Muramatsu H, Kashiwagi F, Katayama Y, Terashi A. Long-term measurement of cerebral blood flow and metabolism in a rat chronic hypoperfusion model. Clin Exp Pharmacol Physiol. 2003;30(4):266-272.

43. Shibata M, Ohtani R, Ihara M, Tomimoto H. White matter lesions and glial activation in a novel mouse model of chronic cerebral hypoperfusion. Stroke. 2004;35(11):2598-2603

44. Miki K, et al. Intensity of chronic cerebral hypoperfusion determines white/gray matter injury and cognitive/motor dysfunction in mice. J Neurosci Res. 2009;87(5):1270-1281.

45. Greenberg JH, LoBrutto C, Lombard KM, Chen J. Postmortem diffusion of autoradiographic blood flow tracers. Brain Res. 1999;842(1):184-191.

46. Lacombe P, Oligo C, Domenga V, Tournier-Lasserve E, Joutel A. Impaired cerebral vasoreactivity in a transgenic mouse model of cerebral autosomal dominant arteriopathy with subcortical infarcts and leukoencephalopathy arteriopathy. Stroke. 2005;36(5):1053-1058.

47. Nielsen LB, et al. Human apolipoprotein B transgenic mice generated with 207- and 145-kilobase pair bacterial artificial chromosomes. Evidence that a distant 5 '-element confers appropriate transgene expression in the intestine. J Biol Chem. 1997;272(47):29752-29758.

48. McCormick SP, Liu CY, Young SG, Nielsen LB. Manipulating large insert clones for transgenesis. Methods Mol Biol. 2003;209:105-123.

49. Franklin KBJ, Paxinos G, eds. The Mouse Brain in Stereotaxic Coordinates (2nd edition). San Diego, CA: Elsevier Academic Press; 2001.

50. Jay TM, Lucignani G, Crane AM, Jehle J, Sokoloff L. Measurement of local cerebral blood flow with [14C]iodoantipyrine in the mouse. J Cereb Blood Flow Metab. 1988;8(1):121-129.

51. Gaertner RF, Wyss-Coray T, Von Euw D, Lesne $\mathrm{S}$, Vivien D, Lacombe P. Reduced brain tissue perfusion in TGF-beta 1 transgenic mice showing Alzheimer's disease-like cerebrovascular abnormalities. Neurobiol Dis. 2005;19(1-2):38-46.

52. Frengen E, et al. Modular bacterial artificial chromosome vectors for transfer of large inserts into mammalian cells. Genomics. 2000;68(2):118-126. 